\title{
Passive Scalar Diffusion in the Near Field Region of Turbulent Rectangular Submerged Free Jets
}

\section{Andrea Boghi ${ }^{a}$, Ivan Di Venuta ${ }^{\mathrm{b}}$, Fabio Gori ${ }^{\mathrm{b}, *}$}

a School of Water, Energy and Agrifood, Cranfield University, Cranfield, Bedfordshire MK43 0AL, United Kingdom,

${ }^{b}$ Department of Industrial Engineering, University of Rome "Tor Vergata", Via del Politecnico 1, 00133 Rome, Italy.

*Corresponding Author, gori@uniroma2.it

\begin{abstract}
Jets are a common way to transfer mass among fluids, or from a fluid to a surface. At moderate Reynolds numbers and low turbulent intensities the jet exhibits a Near Field Region (NFR) several diameters long. Numerical results and a theoretical model are presented for the passive scalar diffusion in the NFR of a submerged free jet. Large Eddy Simulations (LES), in the Reynolds number 5000-40,000 and the Schmidt number range 1-100, are performed obtaining the passive scalar fields. Three mathematical models for the passive scalar diffusion are presented; the first one is valid in the NFR, specifically in the Undisturbed Region of Flow (URF), and the other two, obtained under the hypotheses of Tollmien and Görtler momentum spreadings, are valid in the Potential Core Region (PCR). The last two models employ a turbulent Schmidt number inversely proportional to the mean velocity gradient, conclusion obtained by the LES numerical results. The self-similar solutions of the passive scalar show good agreement with the LES results. The wide range of Reynolds and Schmidt numbers investigated gives generality to the results.
\end{abstract}

Keywords: Submerged Rectangular Free Jet; Turbulent Flow; Near Field Region; Undisturbed Region of Flow; Passive Scalar; Large Eddy Simulation; Self-Similarity.

\section{Nomenclature}

D diameter

$f \quad$ instantaneous self-similarity function

$F \quad$ mean self-similarity function

$h \quad$ half-height of the slot

$k \quad$ turbulent kinetic energy

$P \quad$ mean static pressure

$p \quad$ instantaneous static pressure

$r \quad$ particle radius

$S_{i j} \quad$ rate of shear tensor

$t \quad$ time

$U \quad$ mean axial velocity

$u \quad$ instantaneous axial velocity

$V \quad$ mean cross-stream velocity

$v \quad$ instantaneous cross-stream velocity $y \quad$ cross-stream coordinate

Dimensionless parameters

a similarity coefficient

c Tollmien's coefficient

e Görtler's coefficient

$P e=R e \times S c$ Peclet number

$R e=\frac{U_{i n} D_{h}}{v} \quad$ Reynolds number

$S c=\frac{v}{\Gamma} \quad$ Schmidt number

$\mathrm{Sc}_{\mathrm{T}} \quad$ Turbulent Schmidt Number

Ti Turbulent intensity

Greek

$\alpha_{G} \quad$ passive scalar coefficient (Görtler-like)

$\alpha_{T} \quad$ passive scalar coefficient (Tollmien-like) 


$\begin{array}{ll}\dot{\gamma} & \text { shear rate } \\ \Gamma & \text { Passive scalar molecular diffusivity } \\ \Delta & \text { filter width } \\ \zeta & \text { Tollmien's self-similarity variable } \\ \eta & \text { self-similarity variable } \\ \mathrm{K}_{\Phi} & \text { passive scalar coefficient } \\ \mu & \text { dynamic viscosity } \\ v & \text { kinematic viscosity } \\ v_{T} & \text { turbulent viscosity } \\ \xi & \text { Görtler's self-similarity variable }\end{array}$

$\begin{array}{ll}\tau_{i j}^{R} & \text { Reynolds stress tensor } \\ \phi & \text { instantaneous passive scalar } \\ \Phi & \text { mean passive scalar } \\ \psi & \text { instantaneous stream-function } \\ \Psi & \text { mean stream-function }\end{array}$

Subscripts

$h \quad$ hydraulic

in inlet

sgs sub-grid scale

$T \quad$ turbulent

\section{Introduction}

Heat and mass transfer among free jets and between a jet and a solid or liquid surface is controlled by the fluid dynamics of the jet. The Reynolds number (Re) and the turbulence intensity (Ti) have a great influence upon the dispersion of particles or droplets in jets [1-3], and the heat transfer from a jet to a solid surface [45]. In several applications of circular jets it is desirable to have high Reynolds number $(\operatorname{Re}>40,000)$ and turbulence intensities ( $\mathrm{Ti}>5 \%$ ) and the dispersed phase can be described by some simplified solutions, as in the Fully Developed Region (FDR) [6-7]. In other applications, such as micro-jets for drug injection [8-9], and recent welding technologies [10-11], low turbulence intensities $(\mathrm{Ti}<5 \%)$ and moderate Reynolds numbers $(2,000<\operatorname{Re}<20,000)$ are requested. The dispersed phase field appears to be complex, and there is no mathematical model describing it. One aspect, rarely investigated, is the role that molecular diffusion plays in the micro-jets injection. The diffusivity, $\Gamma$, of a particle is proportional to its radius, according to the StokeEinstein relation [12] and the Cunningham empirical equation [13] allows to conclude that typical values of the particle radius, $r \approx 10^{-7}-10^{-5} \mathrm{~m}$, lead to a Schmidt number of air in the range from 1 to 100 .

The evolution of a turbulent rectangular submerged free jet has been widely investigated in the literature in the last decades with the following conclusion. The jet interacts with the stagnant fluid just downstream of the slot exit and two regions of flow are defined [14]: the closest to the slot exit is the Near Field Region (NFR) and the farthest is the Fully Developed Region (FDR). In the NFR, the region of mixing fluid surrounds the potential core region (PCR), where the velocity on the centerline maintains the same value of the slot exit. When the mixing region has penetrated into the centerline, the NFR ends and the FDR begins. Tollmien [15] and Görtler [16] studied theoretically the flow of turbulent rectangular submerged free jets proposing a self-similar evolution of the axial velocity in the PCR and FDR. The equations describing the velocity evolution were confirmed experimentally in [17-21] for the PCR, and in [22-25] for the FDR. 
In the last few years the evolution suggested in [14], with two regions of flow, has been revised. In the average flow the NFR can be split into two separate regions: the Undisturbed Region of Flow (URF) and the PCR. The URF, reported experimentally between the slot exit and the PCR [26-28], for moderate Reynolds numbers $(\mathrm{Re}=2000-20,000)$ and low turbulence intensity at the inlet $(\mathrm{Ti}=0 \% \div 5 \%)$, has not been noticed neither commented. Several experiments on rectangular free jets of air, conducted by Gori and coworkers [29-34] in the average flow evolution, enabled to identify the main characteristics of the URF, i.e. that velocity and turbulence remain almost equal to those measured on the slot exit. Numerical investigations have been also able to reproduce the URF since Gori et al. [35] undertake preliminary numerical investigations with the Reynolds Averaged Navier-Stokes (RANS) equations model. The URF has been investigated also with the Large Eddy Simulations (LES) approach, at several Reynolds numbers, in order to verify the occurrence of URF, [36-37]. One of the main achievement of the last two studies was to show that the velocity profile in the URF obeys to a self-similar law, different from those proposed by Tollmien [15] and Görtler [16] for the PCR.

The presence of the URF influences the diffusion of the passive scalar in turbulent jets, which has been widely studied, but only limited to the FDR. Despite recognizing the self-similarity of the passive scalar, a mathematical formulation of its diffusion is still missing. The only exceptions are the studies in the FDR of circular jets [6-7], which constitute the base for the "Gaussian Plume Model" (GPM). Such a model, widely used in environmental applications [38-39], was derived under the hypotheses of constant eddy diffusivities for both the momentum and the passive scalar. The lack of a self-similar solution for the passive scalar spreading is probably due to the difficulties in the modeling of the turbulent Schmidt number.

The present work studies the diffusion of a passive scalar in the NFR of turbulent submerged air jets, issuing from a rectangular nozzle. The Schmidt numbers investigated range from 1 to 100, while the Reynolds numbers from 5000 to 40,000. Since the Peclet number spans between 5000 and 4,000,000 a full threedimensional (3D) simulation is unpractical [40-41]. The present numerical investigation is limited to the NFR and the domain is considered two-dimensional, 2D, which is a common assumption for Direct Numerical Simulations (DNS) [42-46], and LES [47-48] of rectangular jets. Indeed, the near-field transitional mixing in turbulent jets is dominated by 2D large-scale coherent structures, as pointed out in [49]. This approximation has been demonstrated valid also in [37], where the 3D results for the NFR are in good agreement with the 2D results of [36]. In analogy with [36], a top-hat velocity profile is imposed on the slot exit and air is assumed incompressible.

\section{Numerical Method}

\subsection{Governing Equations}

In the LES approach, the governing equations are derived by filtering the Navier-Stokes equations and the filtered variables have the superscript ${ }^{\sim}$. Applying the filtering operation, the mass and momentum equations read: 
$\frac{\partial \tilde{u}_{i}}{\partial x_{i}}=0$

$\frac{\partial \tilde{u}_{i}}{\partial t}+\frac{\partial}{\partial x_{j}}\left(\tilde{u}_{i} \tilde{u}_{j}+\left(\tilde{p}+\frac{2}{3} C_{I} \Delta^{2} \tilde{\dot{\gamma}}^{2}\right) \delta_{i j}-2\left(\frac{1}{\operatorname{Re}}+C_{S} \Delta^{2} \tilde{\dot{\gamma}}\right) \tilde{S}_{i j}\right)=0$

where $u_{i}$ is the velocity vector, $p$ the static pressure, $\delta_{i j}$ the identity tensor, $S_{i j}$ the rate of shear tensor

$$
\tilde{S}_{i j}=\frac{1}{2}\left(\frac{\partial \tilde{u}_{i}}{\partial x_{j}}+\frac{\partial \tilde{u}_{j}}{\partial x_{i}}\right)-\frac{1}{3} \frac{\partial \tilde{u}_{k}}{\partial x_{k}} \delta_{i j}
$$

The filtered shear rate is

$\tilde{\dot{\gamma}}=\sqrt{2 \tilde{S}_{i j} \tilde{S}_{i j}}$

with the filter width given by

$\Delta=\sqrt{\Delta_{m} \Delta_{m}}$

being $\Delta_{k}$ the box filter width in the m-th direction. The constants $C_{I}, C_{S}$ are calculated through the dynamic Smagorinsky model, [50]. As far as the passive scalar diffusion is concerned, the conservation equation is

$$
\frac{\partial \tilde{\phi}}{\partial t}+\frac{\partial}{\partial x_{j}}\left(\tilde{\phi} \tilde{u}_{j}-\left(\frac{1}{P e}+\frac{C_{S} \Delta^{2} \tilde{\dot{\gamma}}}{S c_{s g s}}\right) \frac{\partial \tilde{\phi}}{\partial x_{j}}\right)=0
$$

Unlike the coefficients $C_{I}, C_{S}$ the sub-grid Schmidt number has not been evaluated dynamically but it has been fixed to 1 .

\subsection{Computational details}

The LES numerical simulations are obtained with the finite-volume solvers pisoFoamPS, developed for the present study and implemented in the open-source code OpenFOAM. PisoFoamPS is a transient solver for incompressible, turbulent flow, using the PISO algorithm, which couples the Navier-Stokes equations and the passive scalar transport. The computational grids are generated with blockMesh, the OpenFOAM utility for mesh generation. The geometry is made by a rectangular box, 3 hydraulic diameters long in the vertical direction and 6.5 in axial direction. Some uniform grids are used with the stencil decreasing for the increasing $R e$ and $S c$ in order to have $\left(S c / S c_{s g s}\right) \Delta^{2} \operatorname{Re} \cdot \max \left(C_{S} \tilde{S}\right)<0.1$ for all the cases. This is done to insure that the sub-grid terms do not affect the solution at the jet boundaries. The dimensions of the computational grids for the different cases are listed in Table 1 . 
Table 1: Computational grid details

\begin{tabular}{|l|l|l|l|}
\hline Case & Peclet number & x-direction nodes & y-direction nodes \\
\hline $\mathrm{Re}=5000 \mathrm{Sc}=1$ & $5 \cdot 10^{3}$ & 442 & 262 \\
\hline $\mathrm{Re}=5000 \mathrm{Sc}=100$ & $5 \cdot 10^{5}$ & 1768 & 1048 \\
\hline $\mathrm{Re}=10000 \mathrm{Sc}=1$ & $10^{4}$ & 884 & 524 \\
\hline $\mathrm{Re}=10000 \mathrm{Sc}=100$ & $10^{6}$ & 1768 & 1048 \\
\hline $\mathrm{Re}=20000 \mathrm{Sc}=1$ & $2 \cdot 10^{4}$ & 884 & 524 \\
\hline $\mathrm{Re}=20000 \mathrm{Sc}=100$ & $2 \cdot 10^{6}$ & 1768 & 1048 \\
\hline $\mathrm{Re}=40000 \mathrm{Sc}=1$ & $4 \cdot 10^{4}$ & 1768 & 1048 \\
\hline $\mathrm{Re}=40000 \mathrm{Sc}=100$ & $4 \cdot 10^{6}$ & 3536 & 2096 \\
\hline
\end{tabular}

To reproduce the same conditions of $[14,36]$ a top-hat velocity profile is assumed on the slot exit. The walls, above and below the slot, have a thickness of 0.176 hydraulic diameters, while everywhere else, in the $\mathrm{x}$ and $\mathrm{y}$ directions, there is a free boundary, in analogy with [36, 37]. As far as the passive scalar is concerned, a top-hat profile is imposed on the inlet, and a Neumann boundary condition is used everywhere else. The "simple" interpolation filter is chosen for the LES simulation, with an amplitude equal to the cubic root of the cell volume. As far as the time discretization is concerned, a second order backwards scheme is chosen for the time integration, with a time step satisfying the condition $\mathrm{CFL}<0.5$. The numerical scheme adopted for the spatial derivatives is a central second order scheme. The mean turbulent properties are obtained by averaging the instantaneous variables for 100 "flow time", defined as the ratio between the domain length and the axial velocity on the slot exit, after the steady state is reached.

\section{Numerical Results}

The numerical results are presented by showing the relevant fields, and analyzing the properties of the passive scalar profiles shown by the instantaneous and the average numerical results.

\subsection{Instantaneous results}

Figures 1-2 report the instantaneous passive scalar profiles, after 74 flow times, for Reynolds number equal to 5000, 10,000, 20,000, 40,000, and Schmidt number equal to 1 and 100. In the instantaneous flow evolution the URF has been further divided into two different types of flow, [51], the first one named Negligible Disturbances Flow, NDF, and the second one Small Disturbances Flow, SDF. In the NDF the height of the jet flow remains constant with the distance from the slot exit while in SDF the height oscillates without forming vortices.

The structure of the passive scalar field is similar in all cases, since it mimics the instant vorticity field shown in [36-37]. The NDF and SDF, present just downstream of the slot exit, are eddy-free regions, where the velocity profile remains almost unaltered compared to the slot exit, [26-28], and is self-similar [36-37]. 
The NDF and SDF shrink with the increasing Reynolds number, in agreement with [29-37], and almost disappear at $\mathrm{Re}=40,000$, [36-37], allowing the classical two-region description [14]. Downstream the NDF and SDF a region with symmetric vortex-pairs, i.e. the Potential Core Region (PCR), appears at the interface with the stagnant air. The velocity profile spreads more quickly compared with the NDF and SDF, but the velocity at the centerline remains constant. The symmetry of the vortex-pairs is typical of the turbulent flow, whereas, in the laminar regime, the flow field oscillates around the centerline [51]. With the increase of the axial distance the vortices growth occupying the entire thickness of the jet, where the velocity on the centerline diminishes and the PCR ends. After the PCR, the last region of flow, i.e. the FDR, where the vortex-stretching occurs, appears. The FDR is not observable in the present results because the domain is $2 \mathrm{D}$. The lack of vortexstretching, and the consequent absence of the FDR, in the 2D modeling, does not affect the validity of the results because the present work studies the passive-scalar diffusion in the NFR, where the vortex-stretching is not important, as pointed out in [42].

The turbulent stresses in the NDF and SDF are negligible, in comparison to the viscous ones, at the interface between jet and stagnant air, as shown in [36-37]. The NDF and SDF are present in all the numerical simulations, with the same characteristics regardless of the $S c$ number. In the following the differences at the interface, due to the different molecular diffusivity of the passive scalar, are going to be discussed. As far as the Kelvin-Helmholtz Instability (KHI) appears, the mixing begins and the NDF and SDF disappear. The stagnant air is entrained into the vortices and the passive scalar diffuses in these structures.

The diffusion of the passive scalar in the vortices is affected by the value of the $S c$ number. Comparing Fig. 1 with 2, it is evident that the passive scalar contours, in each eddy, are sharper at higher Schmidt numbers. However, it affects the large eddies more than the small ones, as evidenced by Fig. 2, which presents the results for $S c=100$. The first two "mushroom" structures of Figs. 2a, $2 \mathrm{~b}$ present a very sharp passive scalar transition. Nevertheless, from the third vortex pair onwards the transition becomes milder, and the passive scalar contours for the two $S c$ numbers appear very similar. This is because the diffusion in this structure is affected by turbulence, and the molecular mixing is negligible. In conclusion, the molecular diffusion seems to be important only for the large, initial structures, because they are unaffected by turbulence. 


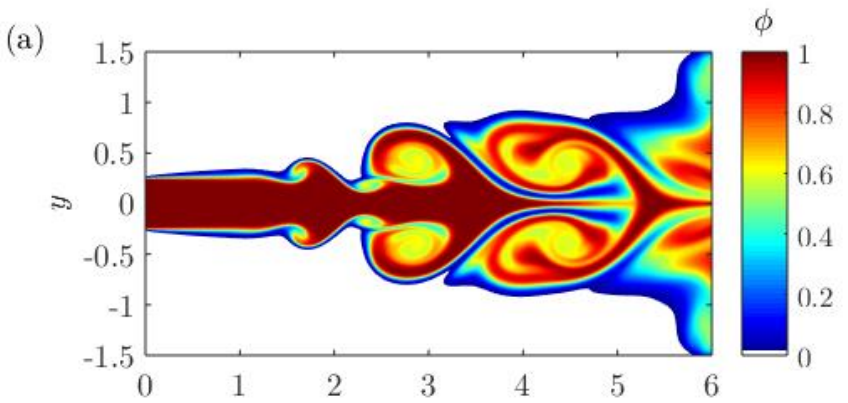

(b)

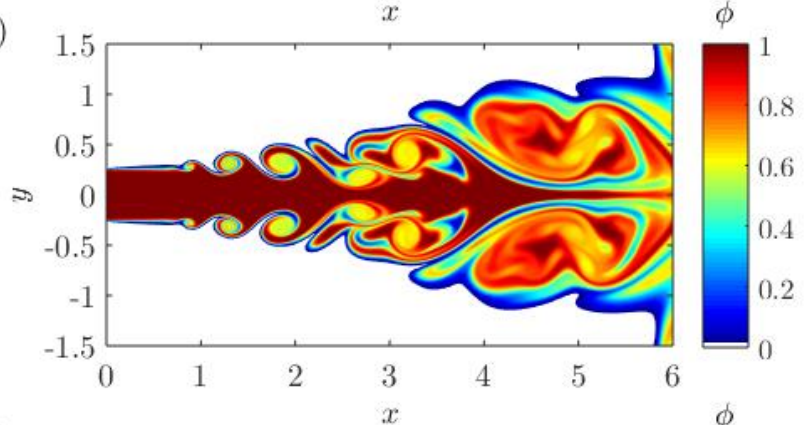

(c)
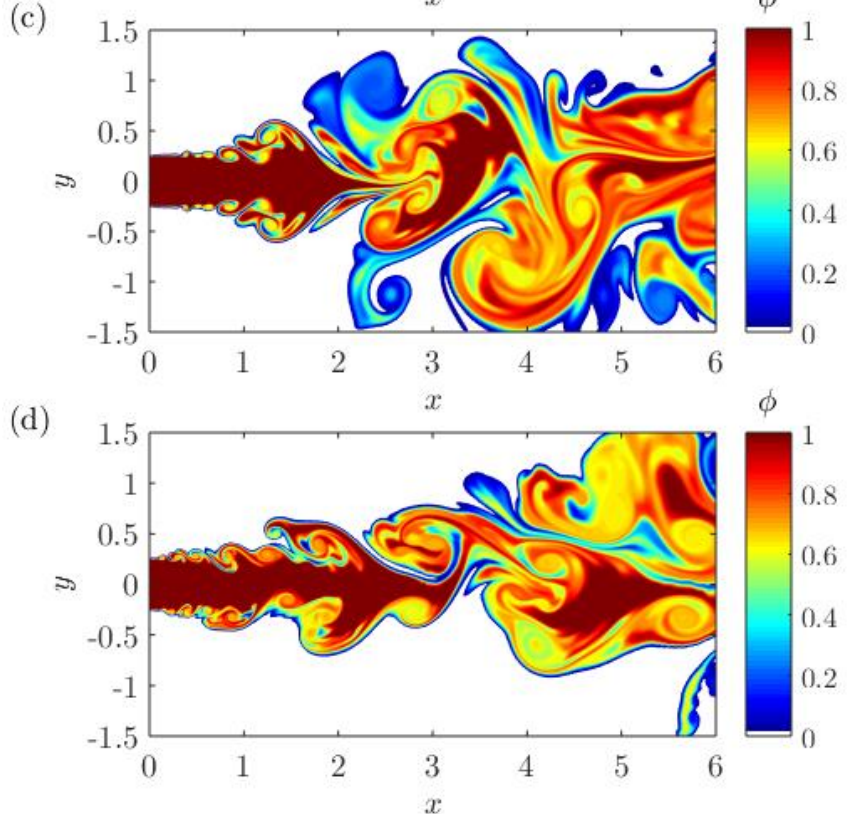

Figure 1: Instant passive scalar contours for $\mathrm{Sc}=1$. (a) $\operatorname{Re}=5000$; (b) $\operatorname{Re}=10,000$; (c) $\operatorname{Re}=20,000$; (d) $\operatorname{Re}=40,000$. 
(a)

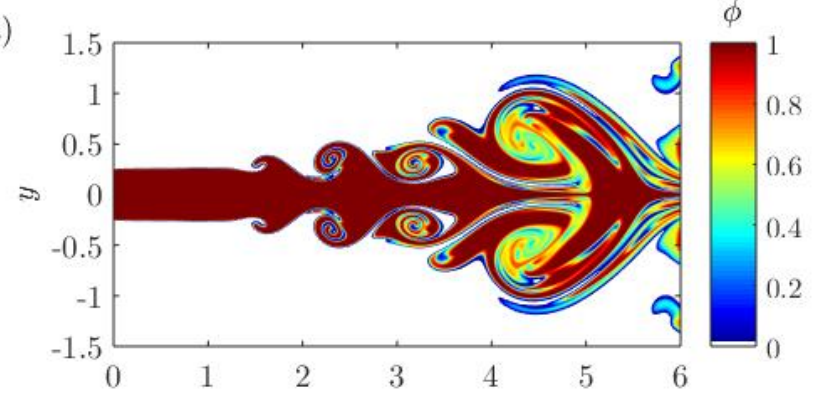

(b)

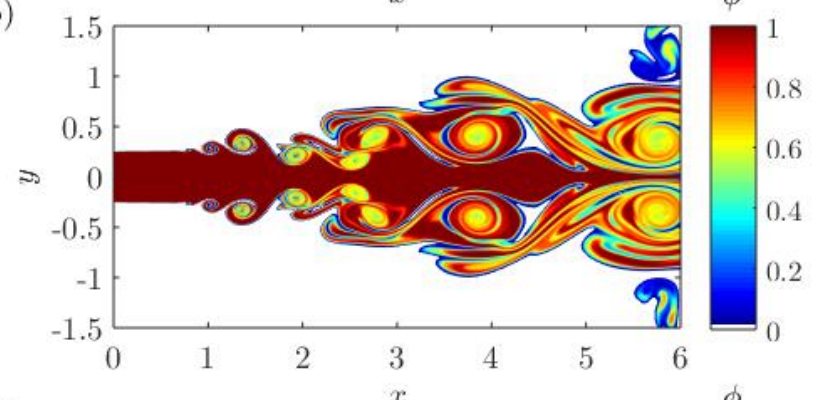

(c)

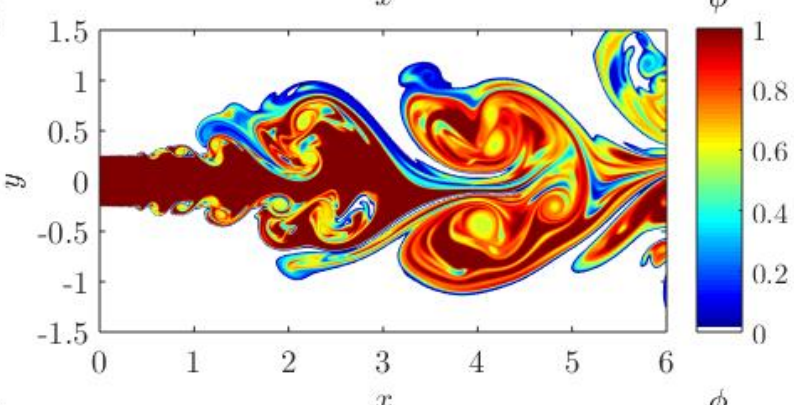

(d)

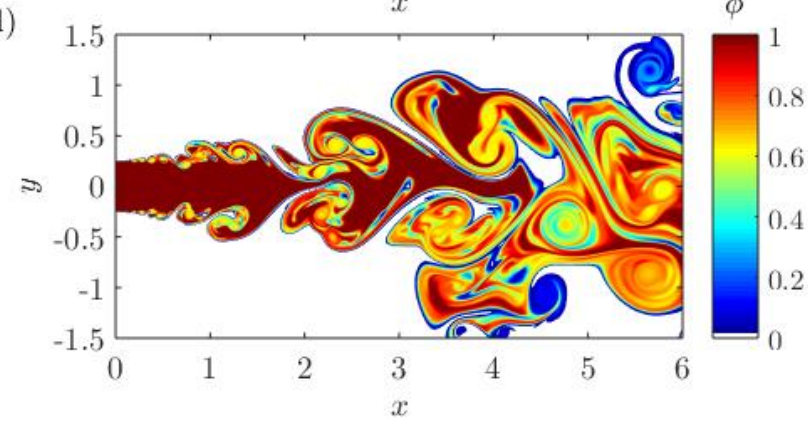

Figure 2: Instant passive scalar contours for $\mathrm{Sc}=100$. (a) $\mathrm{Re}=5000$; (b) $\operatorname{Re}=10,000$; (c) $\operatorname{Re}=20,000$; (d) $\operatorname{Re}=40,000$.

\subsection{Average results}

Mean, variance and turbulent passive scalar flux components, are reported in Figs. 3-6, for the same Reynolds and Schmidt numbers of Figs. 1 and 2. These variables are mostly affected by the Reynolds number.

Figures 3 and 4 show clearly the presence of a region, the URF, where the passive scalar profile remains unaltered compared to that on the slot exit. The length of the URF decreases with the increasing Reynolds number, being approximately equal to $x=2 h$ at $\operatorname{Re}=5,000, x=1.4 h$ at $\operatorname{Re}=10,000, x=0.6 h$ at $\operatorname{Re}=$ 20,000 and $x=0.1 h$ at $\mathrm{Re}=40,000$. Downstream of the URF, the spread of the mean passive scalar increases considerably, evidencing a triangular region, with the tip on the centerline, where $\Phi=1$, which is typical of 
the PCR. An extensive mixing layer, about $2 h$ thick, is present above and below the jet axis, where the passive scalar profile changes gradually. A closer look at the mixing layer identifies three different regions: the central one, where $\Phi=1$, a thin low-turbulence sub-layer, where the passive scalar diffuses slowly and the average value of the mean passive scalar is around 0.85 , and a thick high-turbulence sub-layer, where the passive scalar diffuses quickly. The end of the PCR is not clearly visible, due to the lack of vortex-stretching. For this reason, at $\operatorname{Re}=5,000$ and 10,000 the mean passive scalar, degrading at the center of the jet, is not visible, but it occurs at $\mathrm{Re}=20,000$, although the triangular region is rather concave and longer, and at $\mathrm{Re}=40,000$, where the PCR is more triangular and the tip shorter. The deformed triangular region in the PCR is due to the lack of FDR, which makes the PCR longer.

The main differences between Fig. 3 and 4 concern the URF because the mean passive scalar profile in the mixing region is independent of the molecular diffusivity. This property is analyzed in detail in the following sections. The interface between the URF and stagnant fluid has a milder profile at $\mathrm{Sc}=1$, while the transition is very steep at $\mathrm{Sc}=100$. 


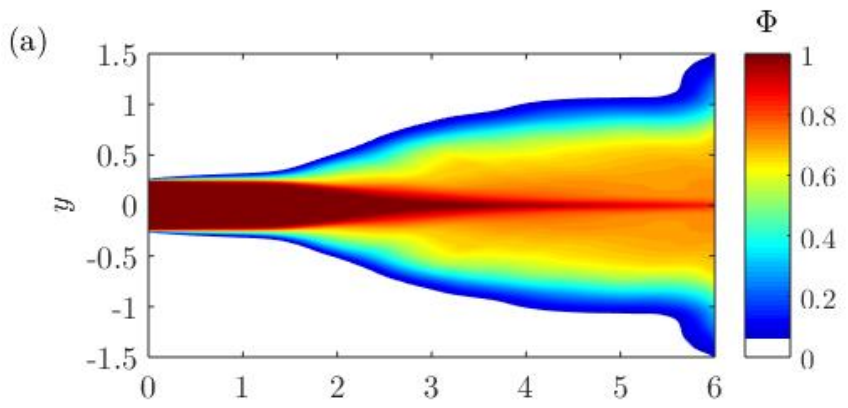

(b)

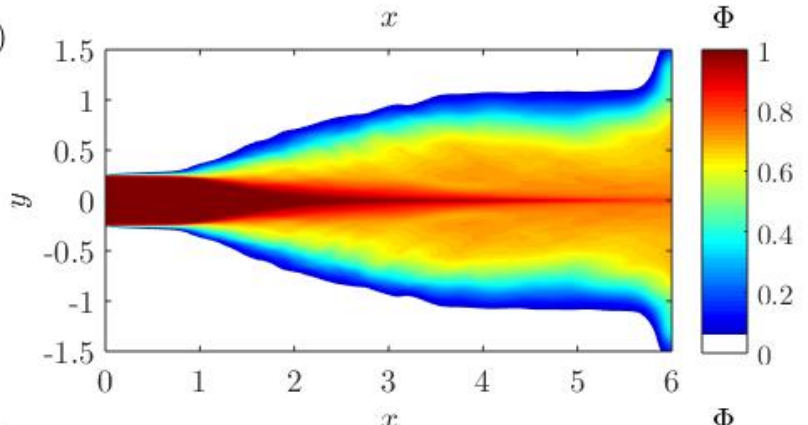

(c)
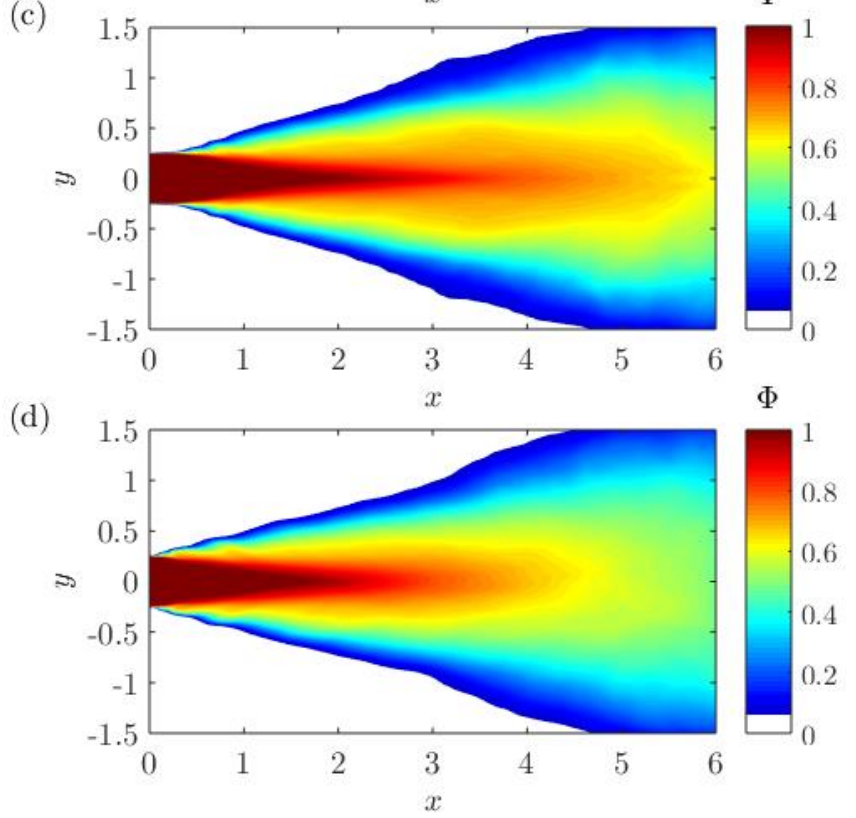

Figure 3: Mean passive scalar contours for $\mathrm{Sc}=1$. (a) $\operatorname{Re}=5,000$; (b) $\operatorname{Re}=10,000$; (c) $\operatorname{Re}=20,000$; (d) $\operatorname{Re}=40,000$. 
(a)

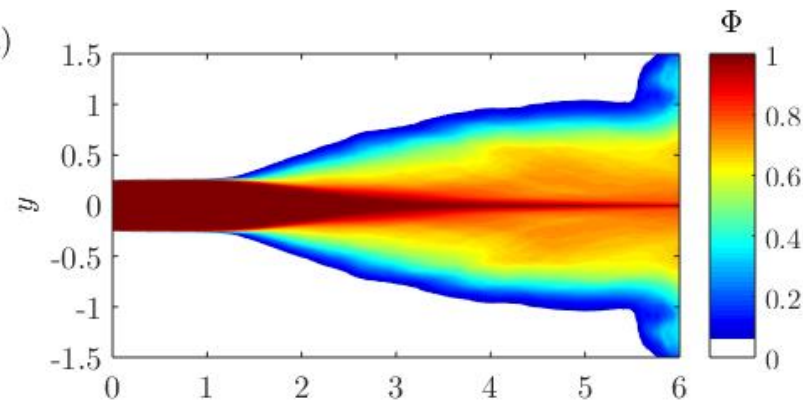

(b)
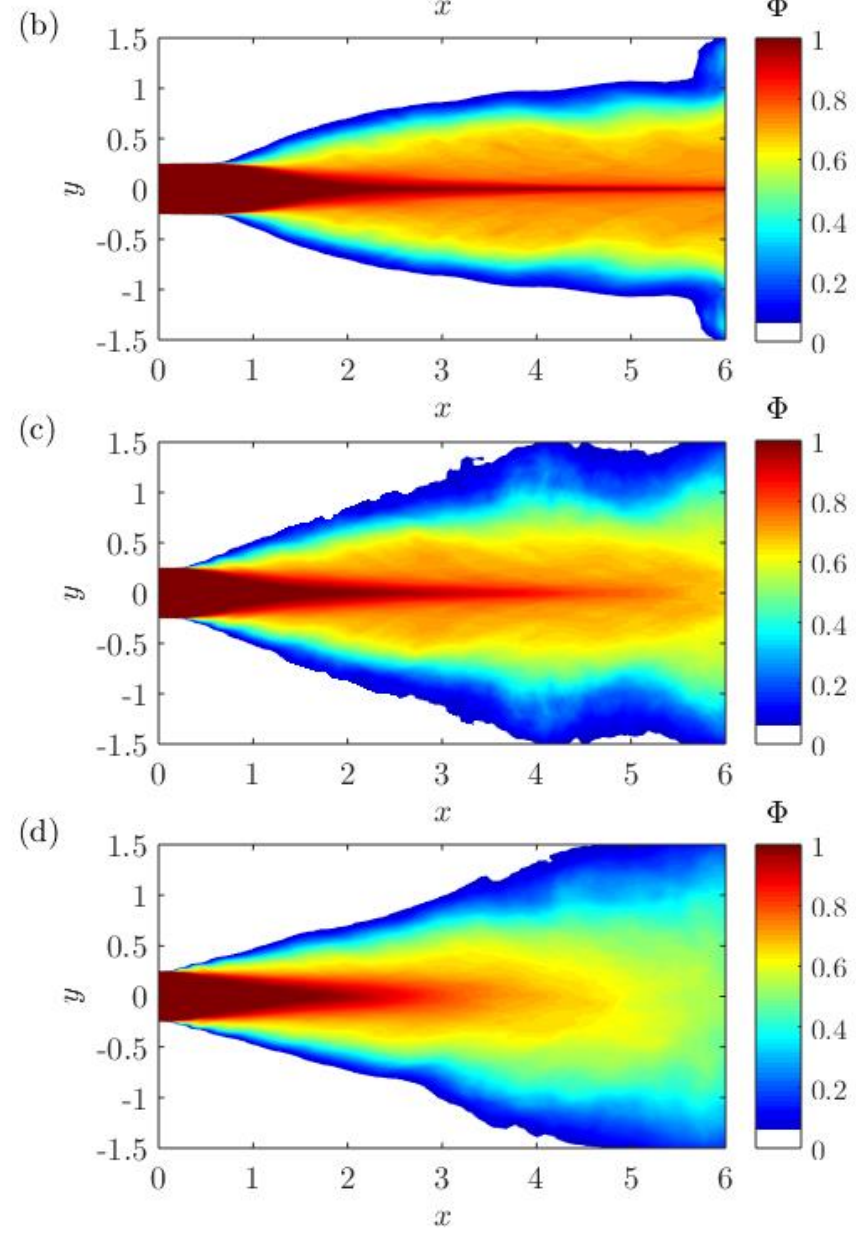

Figure 4: Mean passive scalar contours for $\mathrm{Sc}=100$. (a) $\operatorname{Re}=5,000$; (b) $\operatorname{Re}=10,000$; (c) $\operatorname{Re}=20,000 ;$ (d) $\operatorname{Re}=40,000$.

Figures 5-6 show the contours of the passive scalar variance, $\overline{\phi^{\prime 2}}$. The numerical results show the URF, a region just downstream of the slot exit, where the passive scalar variance is essentially zero, and whose length decreases with the increasing Reynolds number. After the URF, the central triangular region, PCR, shows no passive scalar fluctuations. The passive scalar fluctuations appear in the free shear layer, in agreement with the linear theory on KHI [52], where a low wave number perturbation is enough to trigger the instabilities at high Reynolds number. The passive scalar variance in the PCR assumes slightly higher values for $\mathrm{Sc}=100$ at the interface between jet and stagnant air, which is different from the velocity field, since the correlation of the velocity fluctuations, shown in [36-37], appear to be higher at the center of the mixing layer. The conclusion of the present results is that turbulence affects the passive scalar and the momentum in a 
different way. The passive scalar variance is greater and more uniform, in the far-field, at higher Reynolds numbers.
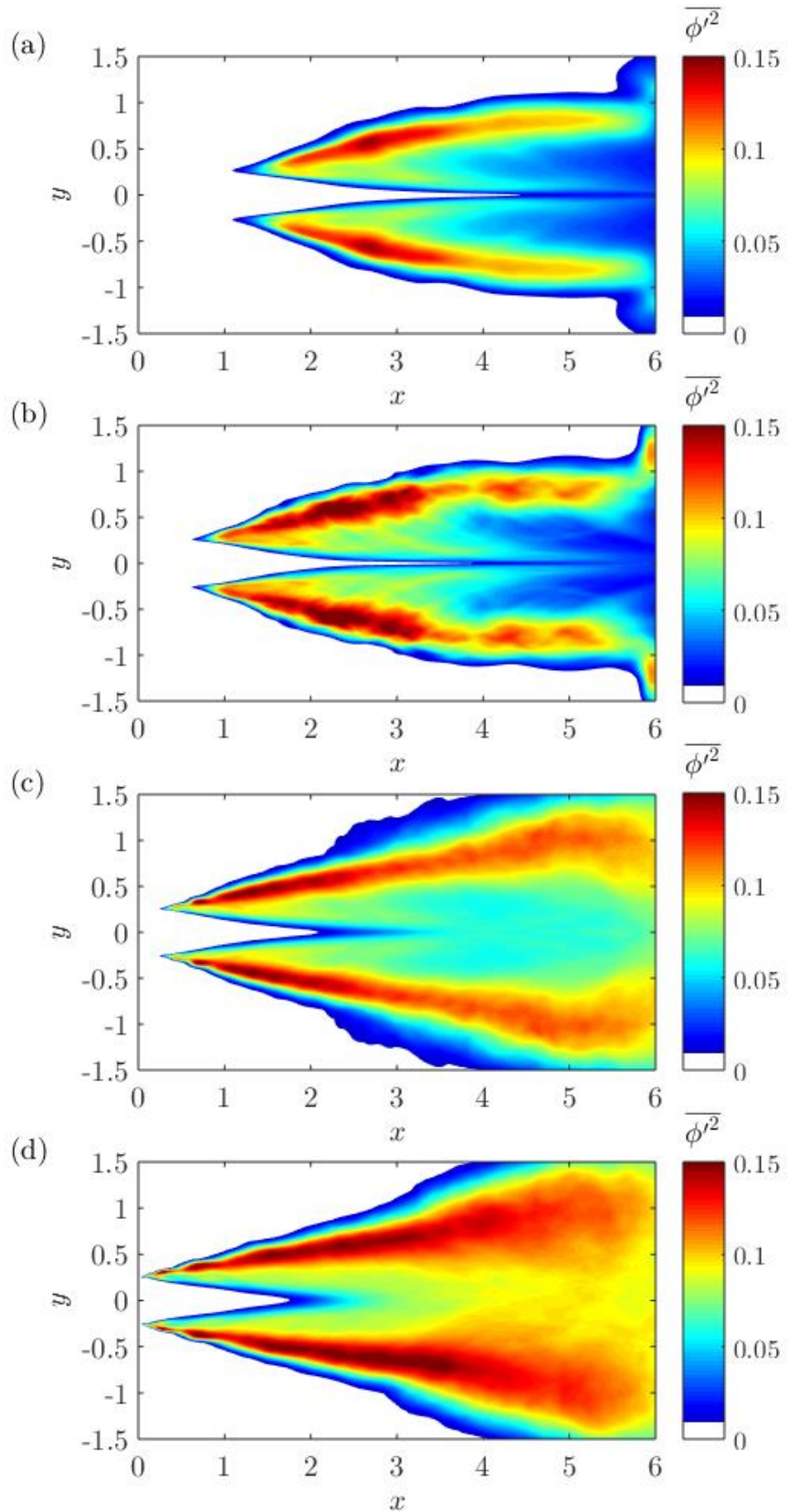

Figure 5: Passive scalar variance contours for $\mathrm{Sc}=1$. (a) $\mathrm{Re}=5,000$; (b) $\mathrm{Re}=10,000$; (c) $\mathrm{Re}=20,000$; (d) $\mathrm{Re}=40,000$. 

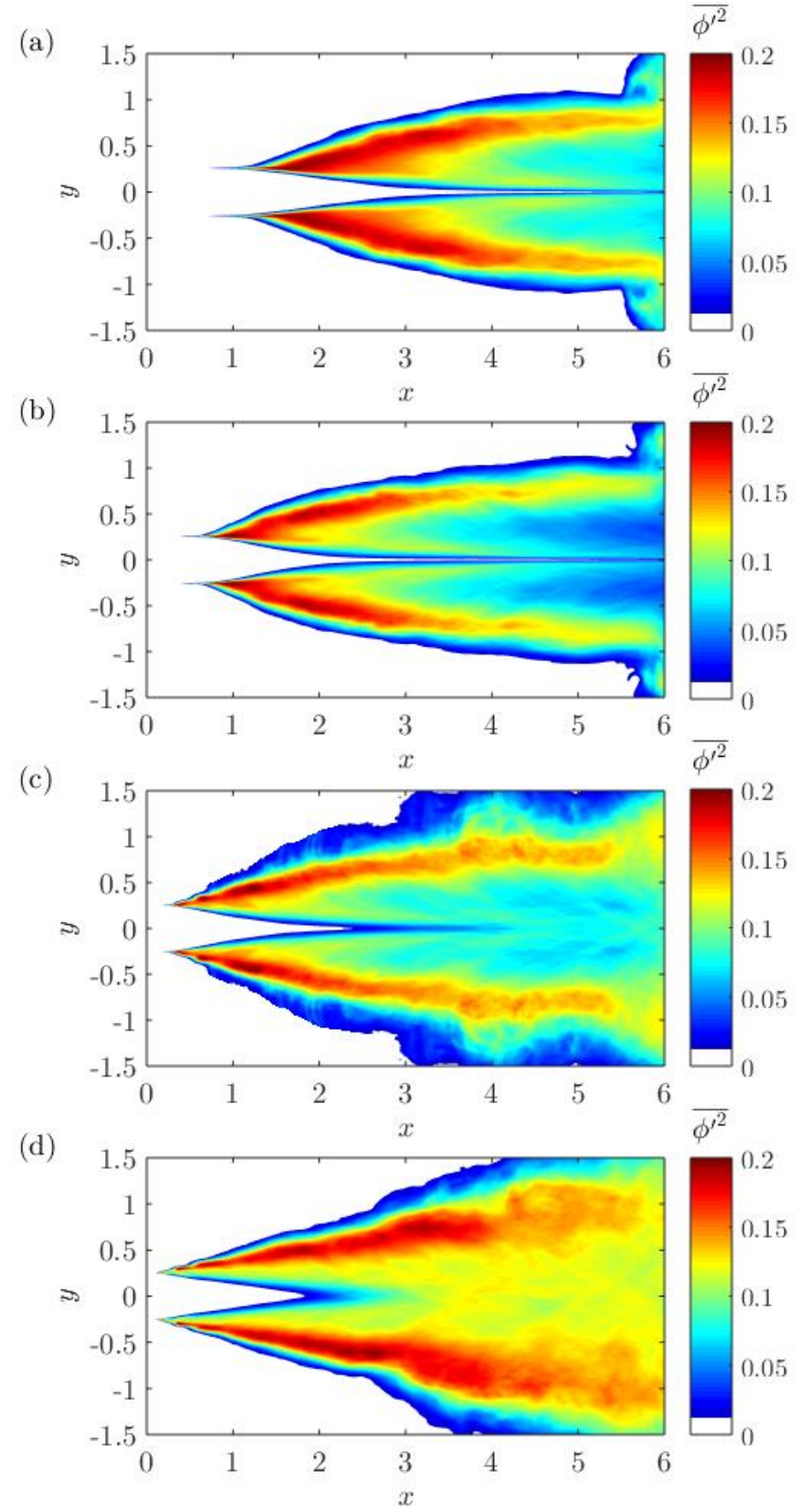

Figure 6: Passive scalar variance contours for $\mathrm{Sc}=100$. (a) $\operatorname{Re}=5,000$; (b) $\operatorname{Re}=10,000$; (c) $\operatorname{Re}=20,000$; (d) $\operatorname{Re}=40,000$.

Figures 7-10 report the components of the axial and transversal turbulent passive scalar fluxes. In both cases, and in analogy with the passive scalar variance, the turbulent fluxes are zero in the URF and in the central region of the PCR.

Figures 7-8 presents the axial component of the turbulent passive scalar flux, which shows a complex pattern because regions of positive and negative values are present. The axial turbulent passive scalar flux assumes lower values than the transversal ones because the lateral diffusion of the passive scalar is predominant. For $\mathrm{Sc}=100$ the axial turbulent passive scalar flux is slightly higher than that for $\mathrm{Sc}=1$.

The axial component of the turbulent passive scalar flux is not proportional to the axial mean passive scalar gradient, as the Boussinesq hypothesis dictates. Downstream of the URF, the passive scalar flux is 
directed towards the two buffer regions, respectively at the boundary with the stagnant air and the PCR, while at the end of the PCR is directed upstream. The pattern at the end of the URF is in first approximation symmetrical with the centerline. It is the result of the instant roller vortices, which move downstream, and are always positive because of the high speed. Towards the end of the PCR the roller vortices are closer to the centerline, as shown by Figs. 1-2, causing a passive scalar flux from the far-field to the inner roller-vortices upstream. Figures 7-8 show also how the axial turbulent passive scalar flux for $\mathrm{Sc}=1$ assumes slightly lower values compared to $\mathrm{Sc}=100$. However, this is not so important since the turbulent flux in the near field is mainly in the transverse direction.

(a)

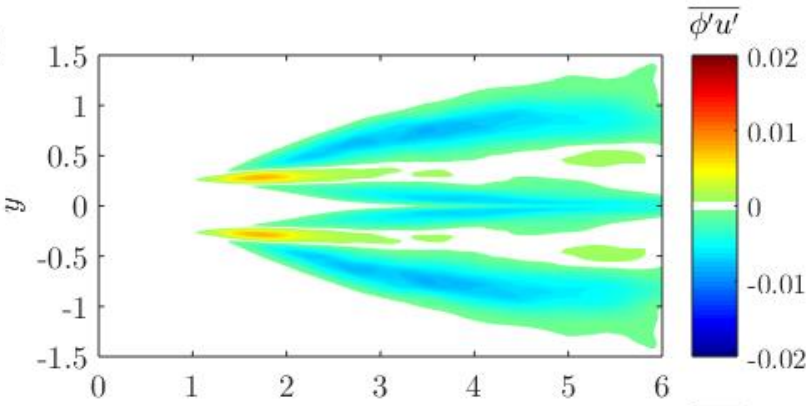

(b)

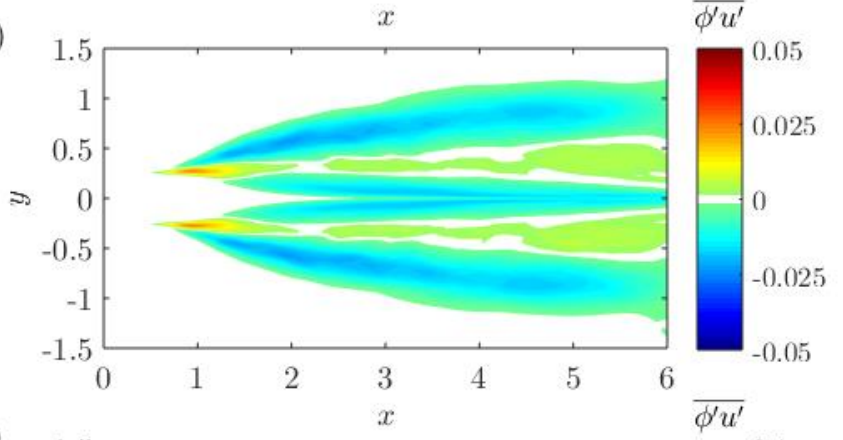

(c)

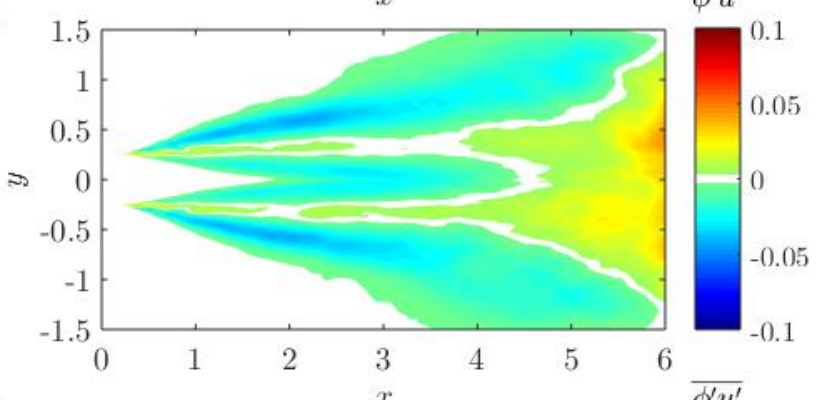

(d)

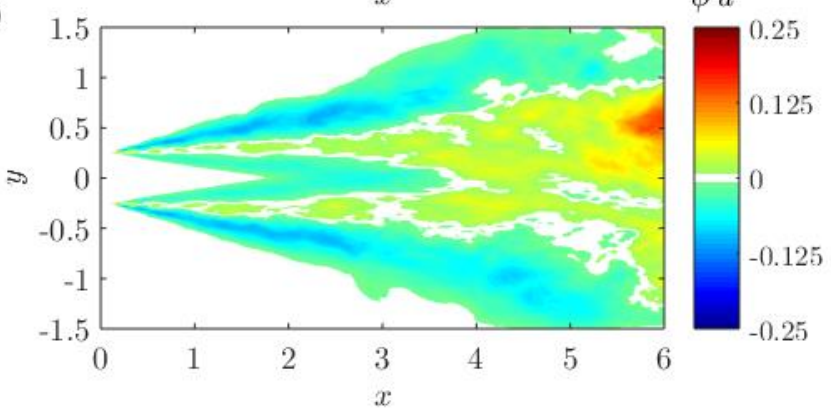

Figure 7: axial component of the turbulent passive scalar flux contours for $\mathrm{Sc}=1$. (a) $\operatorname{Re}=5,000$; (b) $\operatorname{Re}=10,000$; (c) $\operatorname{Re}$ $=20,000 ;$ (d) $\operatorname{Re}=40,000$. 
(a)

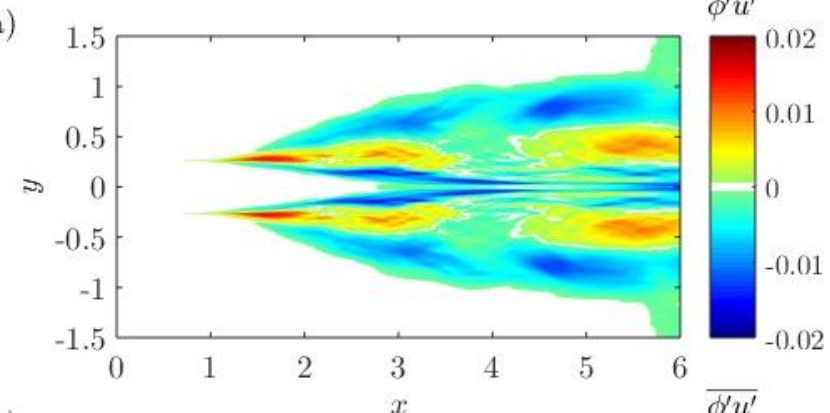

(b)

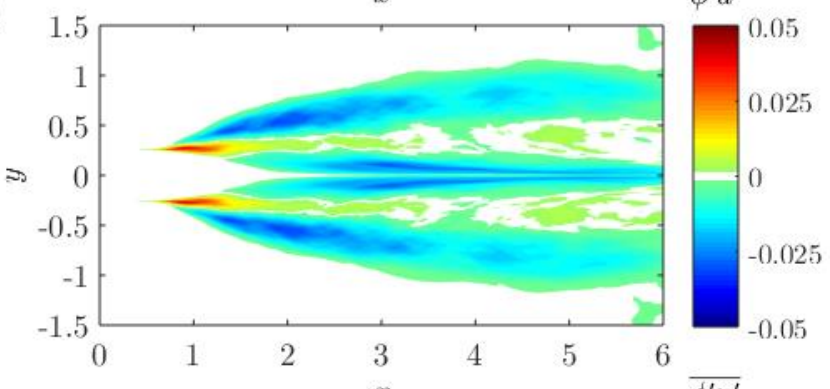

(c)

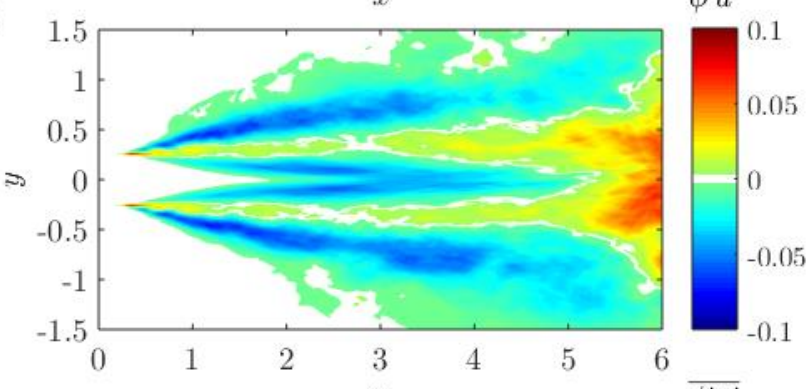

(d)

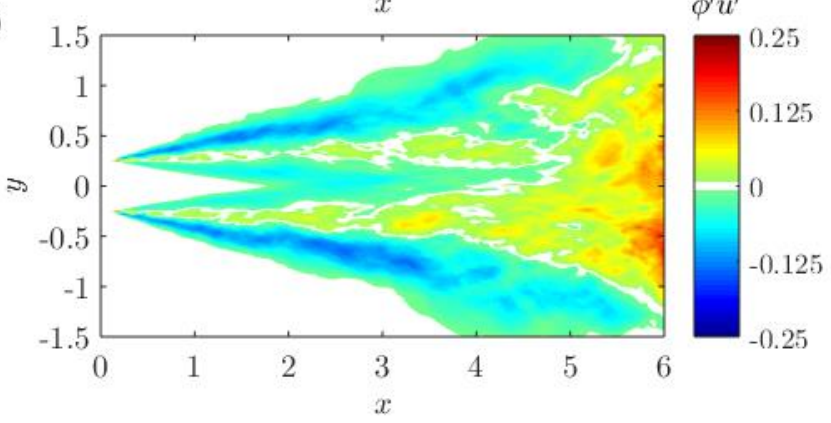

Figure 8: axial component of the turbulent passive scalar flux contours for $\mathrm{Sc}=100$. (a) $\operatorname{Re}=5,000$; (b) $\operatorname{Re}=10,000$; (c)

$$
\operatorname{Re}=20,000 ; \text { (d) } \operatorname{Re}=40,000 \text {. }
$$

Figures 9-10 present the transversal component of the passive scalar turbulent flux, which is predominant in the mixing region surrounding the PCR, despite the presence of the axial flux. The contours of the turbulent passive scalar flux appear to be jagged in analogy with the Reynolds stress contours shown in [37], and, unlike the axial component, is anti-symmetric with respect to the centerline. The transversal component of the passive scalar turbulent flux is directed from the inner part of the jet towards the stagnant air and is always positive in the upper mixing layer, while it is always negative in the lower layer. The transversal component of the passive scalar turbulent flux is zero in the URF and on the center of the PCR, and is responsible for the spreading of the mean passive scalar, shown by Fig. 3-4. Further on, the transversal component of the passive scalar turbulent flux is proportional to the mean passive scalar transversal gradient, 
in agreement to the Boussinesq hypothesis. At $\operatorname{Re}=5,000$ and 10,000 the turbulent transversal flux is antisymmetric, and is higher in the mixing region, but reducing downstream. This reduction is small for Re equal to 20,000 and 40,000 , probably because the turbulence is not affected by the Richardson cascade, due to the lack of vortex-stretching, but, nevertheless, it does not affect the results in the PCR. Furthermore the differences between $\mathrm{Sc}=1$ and $\mathrm{Sc}=100$ are very limited since the vertical turbulent flux assumes similar values.

(a)

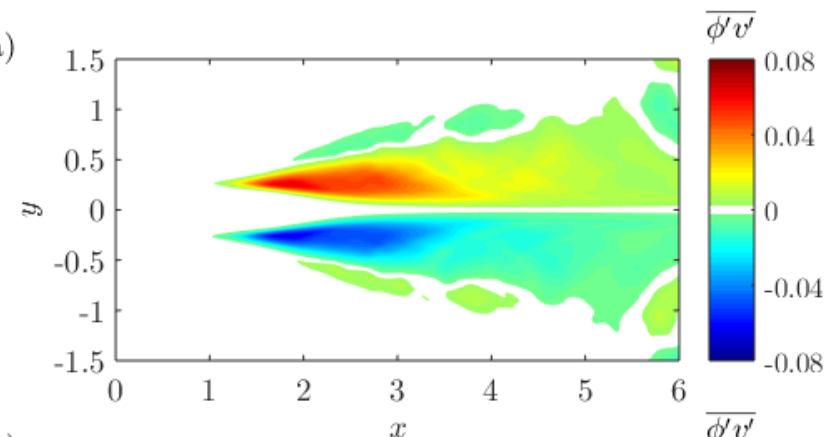

(b)

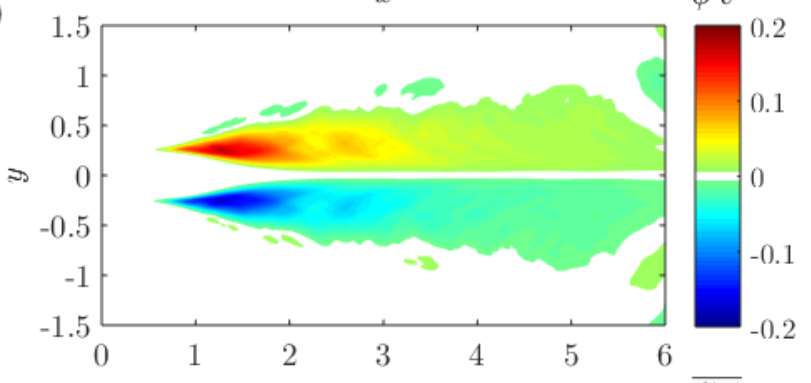

(c)
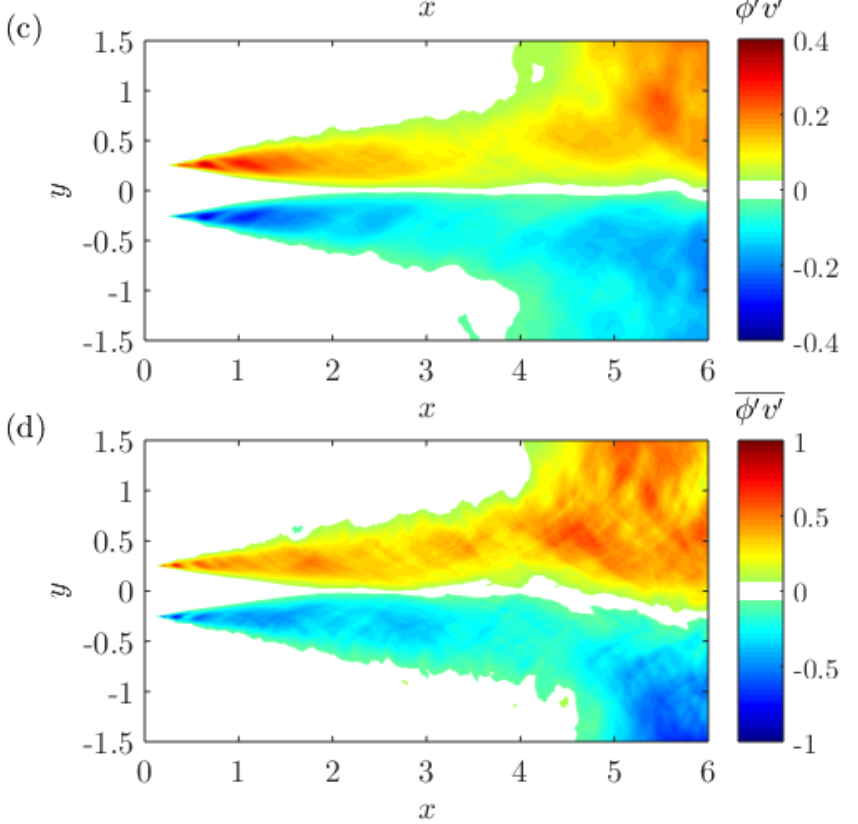

Figure 9: transversal component of the turbulent passive scalar flux contours for $\mathrm{Sc}=1$. (a) $\operatorname{Re}=5,000$; (b) $\operatorname{Re}=10,000$; (c) $\operatorname{Re}=20,000 ;$ (d) $\operatorname{Re}=40,000$. 
(a)

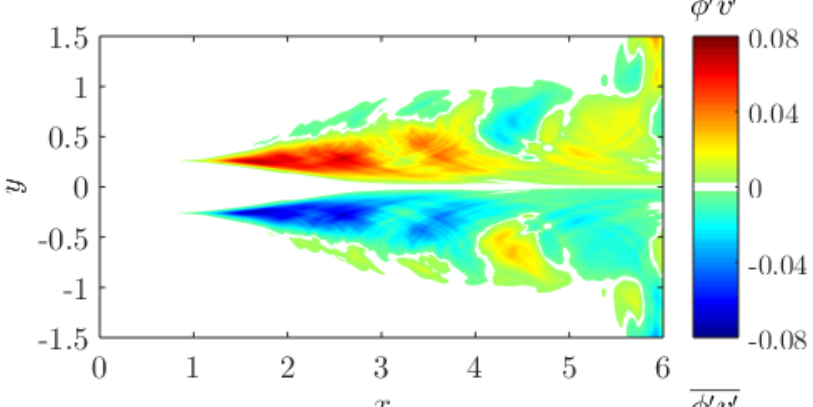

(b)

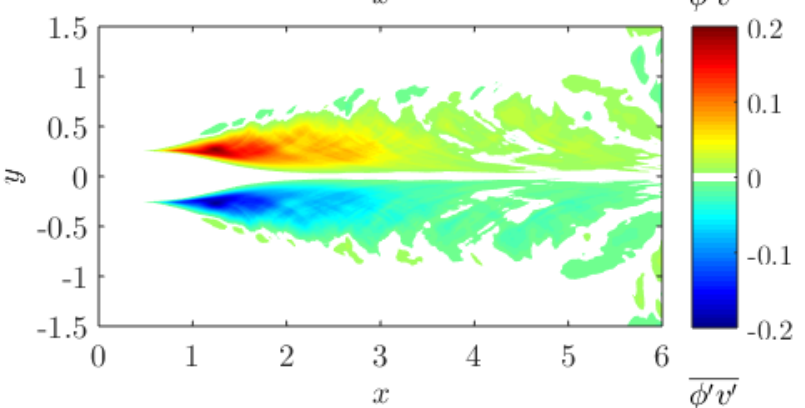

(c)

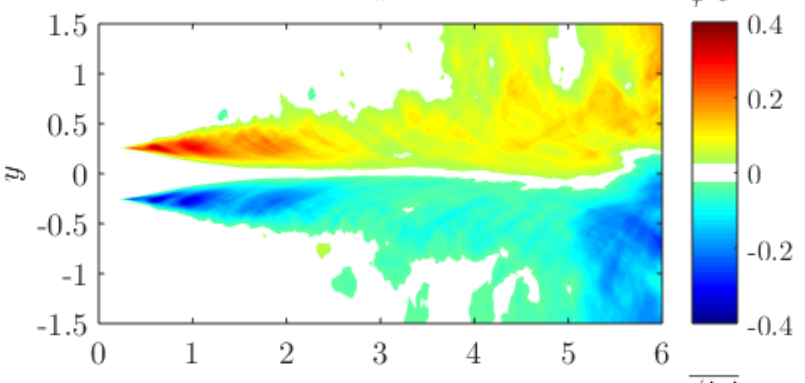

(d)

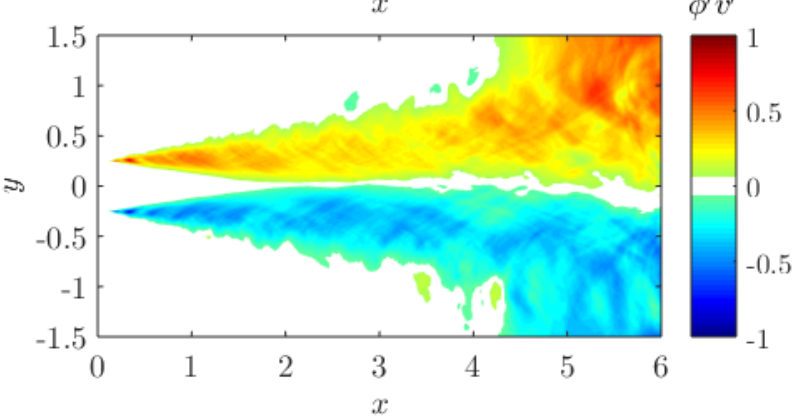

Figure 10: transversal component of the turbulent passive scalar flux contours for $\mathrm{Sc}=100$. (a) $\operatorname{Re}=5,000 ;$ (b) $\operatorname{Re}=$ 10,000; (c) $\operatorname{Re}=20,000 ;$ (d) $\operatorname{Re}=40,000$.

\section{Theoretical mean results}

\subsection{Results in the URF.}

The passive scalar spreading in the URF, in analogy with [36-37], is studied by tracing the iso-passive scalar curves in the (x,y) plane for the Re and Sc numbers investigated. This method is particularly useful to identify discontinuity in the flow field. The evolution is shown only in the upper part of the domain since the average passive scalar profile is symmetric. All the iso-passive scalar curves originate from the point $\left(x_{0}, y_{0}\right)=$ $(0, h)$ since the profile is top-hat on the slot exit. 
Figures 11-12 show the iso-passive scalar curves for the two Schmidt number analyzed, 1 and 100. The analysis of the iso-passive scalar curves reveals the presence of a point, whose axial position is inversely proportional to the Reynolds number, from which the slope of the curves changes rapidly, and marking the end of the URF. Figure 11 shows that the cases with $\mathrm{Sc}=1$ have the thickest interface and is easier to visualize the contours, while, for $\mathrm{Sc}=100$, a steeper transition is present, as shown by Fig. 12.
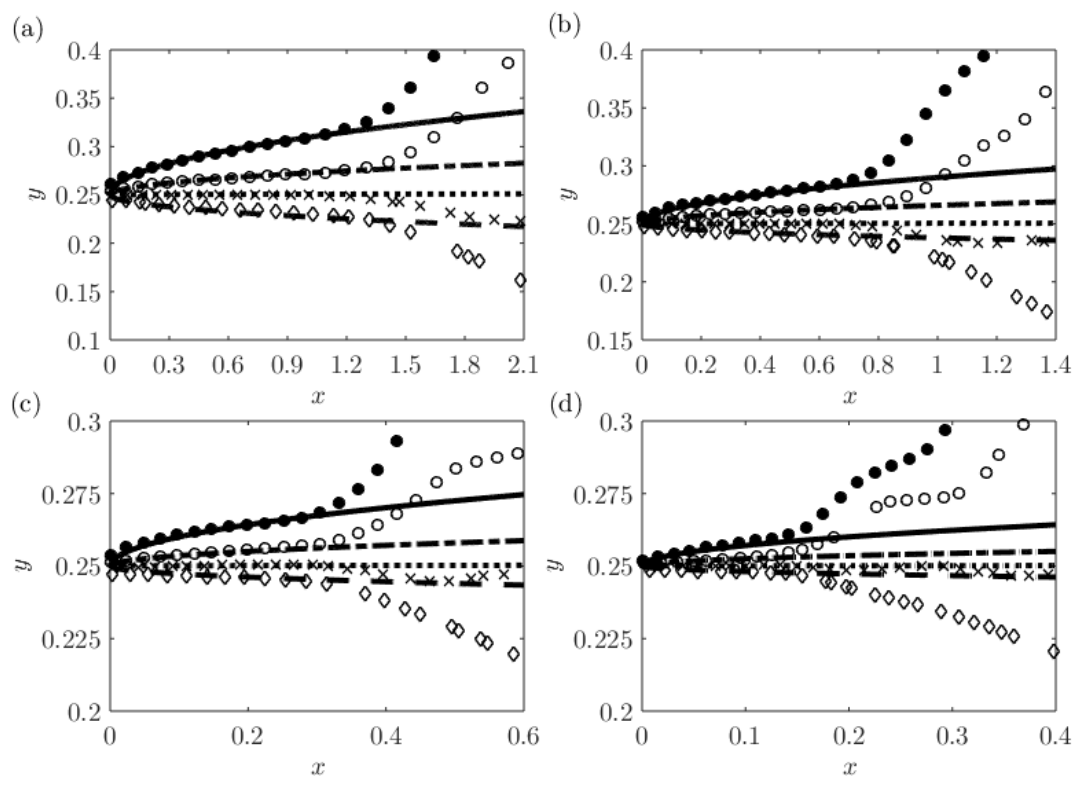

Figure 11: iso-passive scalar curves in URF for $\mathrm{Sc}=1 . \phi=0.1$, data $(\bullet)$, regression $(-) . \phi=0.4$, data $(\circ)$, regression $(-$ .-). $\phi=0.7$, data $(x)$, regression (....). $\phi=0.9$, data $(\diamond)$, regression (---). (a) $\operatorname{Re}=5,000 ;(b) \operatorname{Re}=10,000 ;(c) \operatorname{Re}=$ 20,000; (d) $\operatorname{Re}=40,000$.

(a)

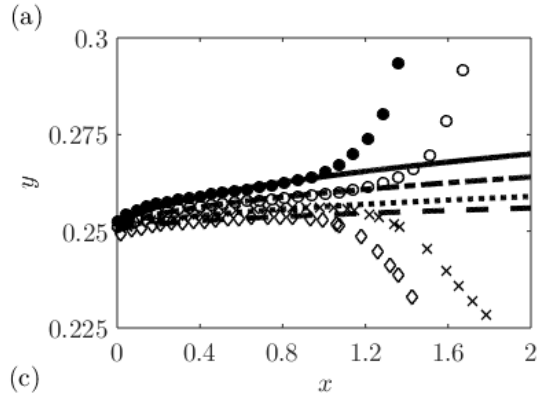

(c)

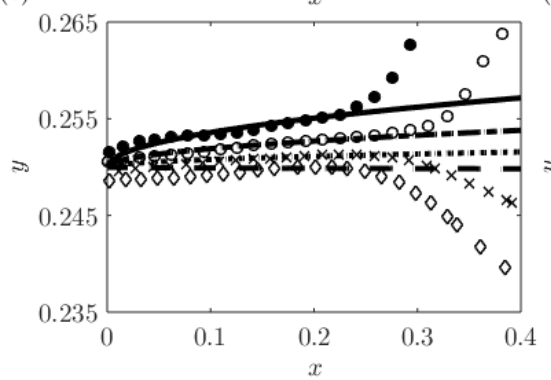

(b)

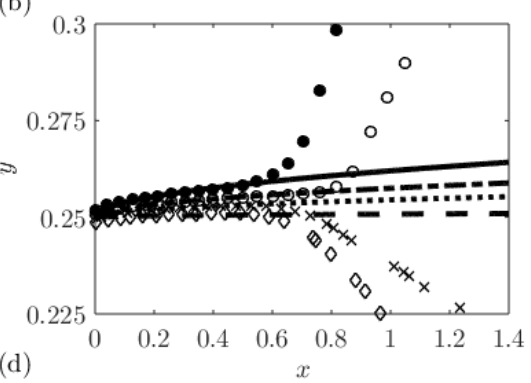

(d)

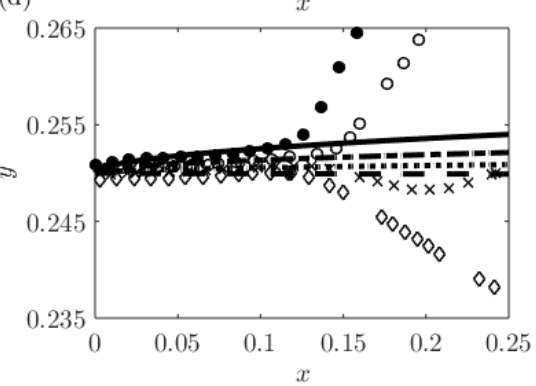


Figure 12: iso-passive scalar curves in URF for $\mathrm{Sc}=100 . \phi=0.1$, data $(\bullet)$, regression $(-) . \phi=0.4$, data (०), regression (-.-). $\phi=0.7$, data (x), regression (....). $\phi=0.9$, data $(\diamond)$, regression (---). (a) $\operatorname{Re}=5,000 ;$ (b) $\operatorname{Re}=10,000 ;(c) \operatorname{Re}=$ 20,$000 ;$ (d) $\operatorname{Re}=40,000$.

Figures 5-10 have shown that the passive scalar variance changes abruptly at a certain distance from the slot exit, i.e. at the interface between the URF and the PCR. The self-similar variable in the URF, in analogy with the axial velocity field [36-37], is given by

$\eta=\eta_{0}+\sqrt{\operatorname{Re}} \frac{(y-h)}{\sqrt{x}}$

where $h$ is the non-dimensional half-slot vertical coordinate, equal to 0.25 and $\eta_{0}=-0.5288$, according to the velocity boundary conditions. The numerical LES results confirm that the self-similar variable in Eq. (7) is constant along the iso-passive scalar curves. The instantaneous stream function reads:

$\psi=\frac{1}{\sqrt{\operatorname{Re}}} \sqrt{x} f(\eta)$

and the self-similar stream function is governed by the following equation:

$\frac{d^{3} f}{d \eta^{3}}+\frac{1}{2} f \frac{d^{2} f}{d \eta^{2}}=0$

The passive scalar budget is analyzed in analogy with the momentum budget in [36-37]. The time derivative is negligible, as well as the turbulent and the axial diffusion, and the passive scalar transport equation reads

$u \frac{\partial \phi}{\partial x}+v \frac{\partial \phi}{\partial y}=\frac{1}{P e} \frac{\partial^{2} \phi}{\partial y^{2}}$

Since the passive scalar is self-similar, as shown in the next Figures 11-12, and, by virtue of Eqs. (79) it is obtained

$\frac{d^{2} \phi}{d \eta^{2}}+\frac{S c}{2} f \frac{d \phi}{d \eta}=0$

which is a second order equation and needs two boundary conditions

$$
\begin{aligned}
& \lim _{\eta \rightarrow+\infty} \phi(\eta)=0 \\
& \lim _{\eta \rightarrow-\infty} \phi(\eta)=1
\end{aligned}
$$


The passive scalar profile is obtained by integrating Eq. (11), with the two boundary conditions of Eqs. (1213)

$$
\phi(\eta)=1-\frac{\int_{-\infty}^{\eta}\left|\frac{d^{2} f}{d \xi^{2}}\right|^{S c} d \xi}{\int_{-\infty}^{+\infty}\left|\frac{d^{2} f}{d \xi^{2}}\right|^{S c} d \xi}
$$

The simplified theory expressed by Eq. (14) is denoted with "U", which stands for "undisturbed", in order to distinguish it from the theoretical results derived in the next section. The modulus of the vertical mass flux per unit area can be obtained from Eq. (14), remembering that $d^{2} f / d \eta^{2}=d u / d \eta$

$$
\left|J_{y}\right|=\frac{1}{P e}\left|\frac{\partial \phi}{\partial y}\right|=\frac{1}{P e} \frac{\left|\frac{\partial u}{\partial y}\right|^{S c} \int_{-\infty}^{+\infty}\left|\frac{d u}{d \xi}\right|^{S c} d \xi}{d \xi}
$$

This equation shows that the mass transfer is function of the velocity gradient and the Schmidt number, and the passive-scalar flux decreases with the increasing Sc number, since the dimensionless velocity gradient is everywhere smaller than 1 .

The passive scalar self-similar profiles, obtained from the solution of Eq. (11), are compared in Figs. 13-14 with the numerical LES results at the Reynolds and Schmidt numbers investigated. The passive scalar profiles are expressed as functions of the self-similar variable, $\eta-\eta_{0}$, and plotted at different axial positions in order to show that the hypothesis of self-similarity is valid. The results at the four Reynolds numbers show a good agreement, especially at the smaller Reynolds number, because of the wider boundary layer. The passive scalar profile is smooth and coincide with the axial velocity profile at $\mathrm{Sc}=1$, Fig. (13). As far as the axial velocity is concerned, the passive scalar profile has a maximum at the center of the jet and decays to zero in the stagnant air. The passive scalar spreading is a function of the Schmidt number. The thickness of the interface between the jet and the stagnant air is inversely proportional to the Schmidt number, in agreement with Eq. (14). For $S c=100$, Fig. 14 shows a good agreement for $\operatorname{Re}=5,000$ and 10,000, while for $\operatorname{Re}=20,000$ and 40,000 the numerical profile appears slightly smoother, probably because of the sub-grid terms. Despite these little discrepancies, the numerical results agree very well with the theory. 
(a)

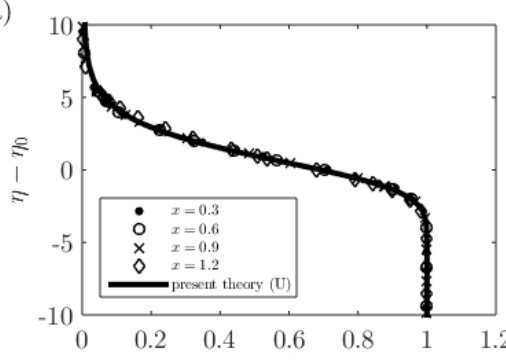

(c)

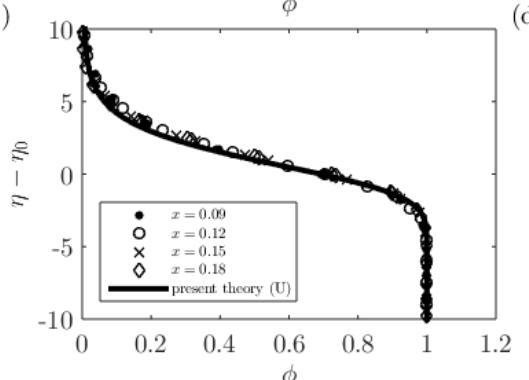

(b)

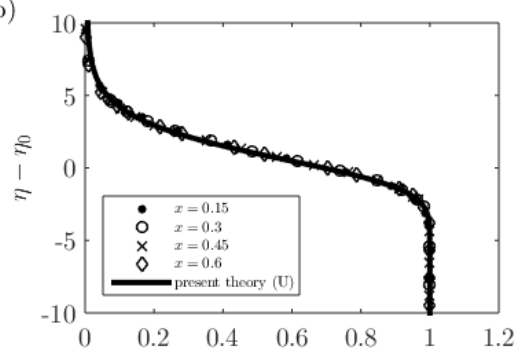

(d)

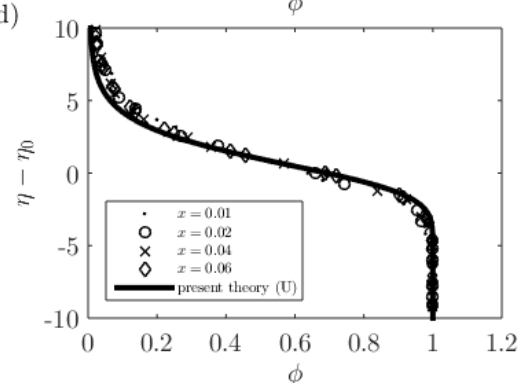

Figure 13: self-similar passive scalar profile in the URF for $\mathrm{Sc}=1$. Numerical and theoretical results. (a) $\operatorname{Re}=5000$; (b) $\operatorname{Re}=10,000 ;$ (c) $\operatorname{Re}=20,000 ;(d) \operatorname{Re}=40,000$.

(a)

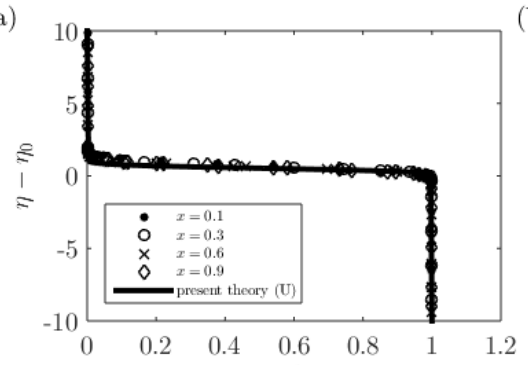

(c)

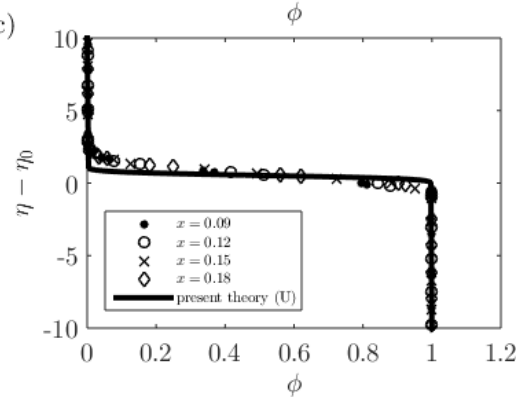

(b)

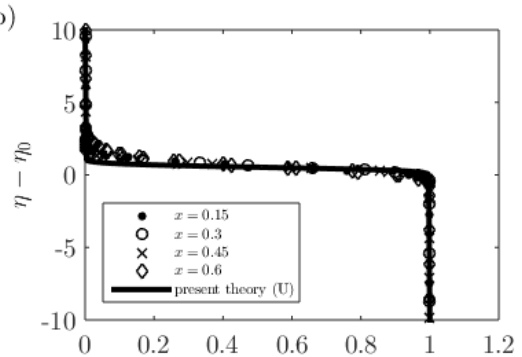

(d)

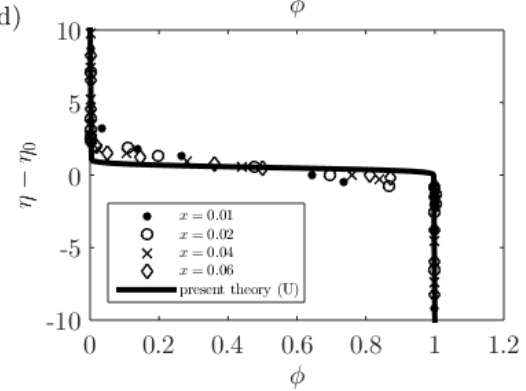

Figure 14: self-similar passive scalar profile in the URF for $S c=100$. Numerical and theoretical results. (a) $\operatorname{Re}=5000$; (b) $\operatorname{Re}=10,000 ;$ (c) $\operatorname{Re}=20,000$; (d) $\operatorname{Re}=40,000$.

\subsection{Results in the PCR.}

The PCR is the region where turbulence, generated at the jet-stagnant air interface, diffuses towards the center. The iso-passive scalar curves are reported for $\mathrm{Sc}=1$ and 100 in Figs. 15 and 16, along with regression. Turbulence is not negligible in the PCR, and the self-similar analysis can be applied only to the mean variables. As Figs. (5-10) suggest, the Schmidt number is not affecting the passive scalar spreading, downstream the URF. 
(a)

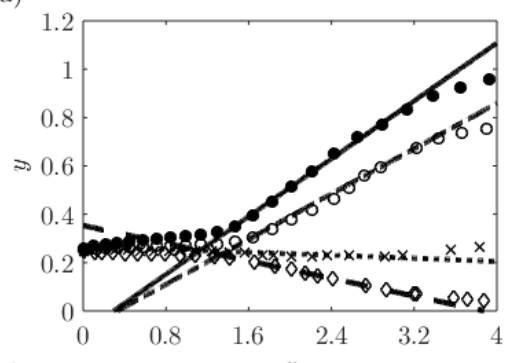

(c)

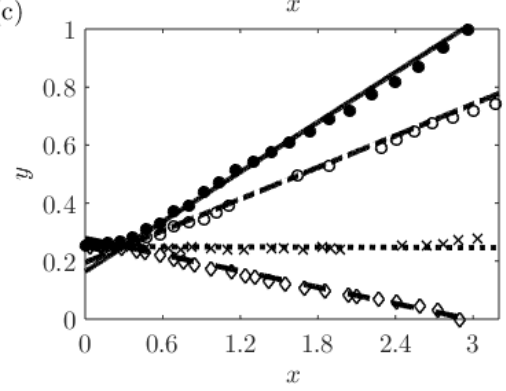

(b)

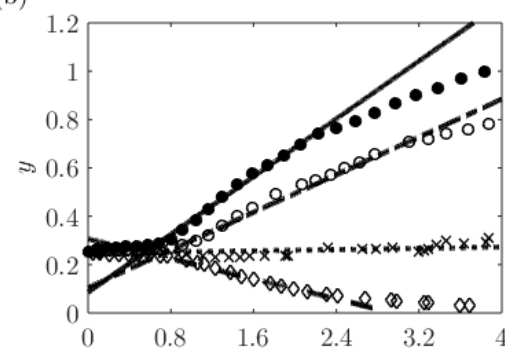

(d)

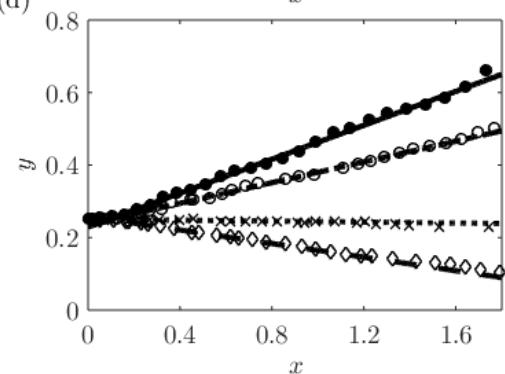

Figure 15: passive scalar iso-curves in PCR for $\mathrm{Sc}=1 . \Phi=0.1$, data $(\bullet)$, regression (-). $\Phi=0.4$, data (०), regression (-.-). $\Phi=0.7$, data (x), regression (....). $\Phi=0.9$, data ( $\diamond$ ), regression (--). (a) $\operatorname{Re}=5000$; (b) $\operatorname{Re}=10,000$; (c) $\operatorname{Re}=$ 20,$000 ;$ (d) $\operatorname{Re}=40,000$.

(a)

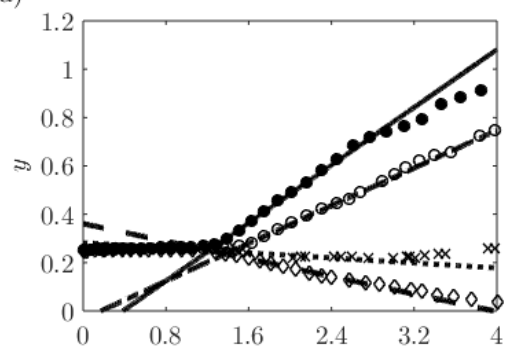

(c)

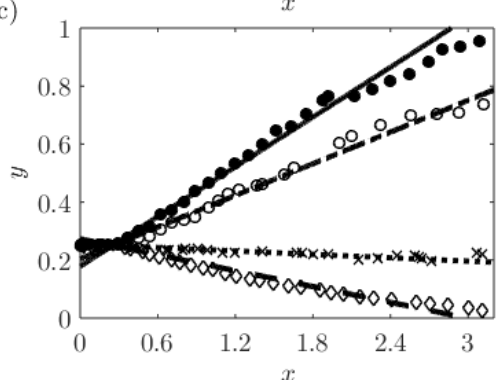

(b)

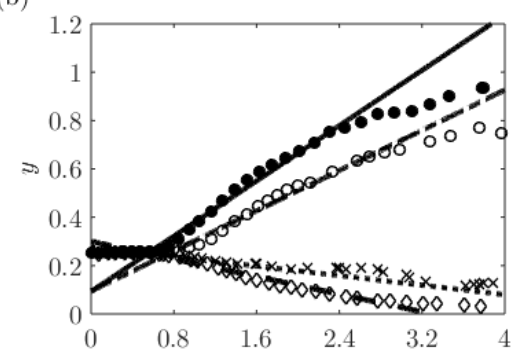

(d)

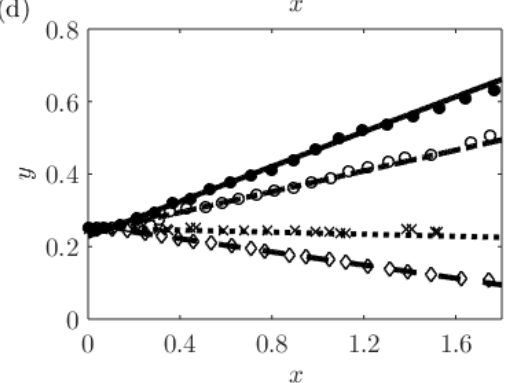

Figure 16: passive scalar iso-curves in PCR for $\mathrm{Sc}=100 . \Phi=0.1$, data $(\bullet)$, regression (-). $\Phi=0.4$, data (०), regression (-.-). $\Phi=0.7$, data (x), regression (....). $\Phi=0.9$, data ( () , regression (--). (a) $\operatorname{Re}=5000$; (b) $\operatorname{Re}=10,000 ;$ (c) $\operatorname{Re}=$ 20,$000 ;$ (d) $\operatorname{Re}=40,000$.

Figures 15-16 show that the passive scalar is constant along the curves, obeying to the following equation 
$\eta=\eta_{0}+\frac{\left(y-h_{0}\right)}{a\left(x-x_{0}\right)}$

with $a=0.13$, in agreement to Tollmien [15] and Görtler [16] for the momentum. The mean stream function reads

$$
\Psi=a\left(x-x_{0}\right) F(\eta)
$$

Two solutions for the self-similar mean stream-function, $F(\eta)$, were found by Tollmien [15] and Görtler [16], whose theories differ by the choice of the self-similar variable and, more importantly, by the turbulent viscosity modelling.

\section{Tollmien [15] proposed}

$v_{T}=c^{2}\left(x-x_{0}\right)^{2}\left|\frac{\partial U}{\partial y}\right|$

which, with the following choice of the self-similarity variable

$$
\zeta=\frac{a\left(\eta-\eta_{0}\right)}{\sqrt[3]{2 c^{2}}}
$$

leads to

$$
\frac{d}{d \zeta}\left(\left|\frac{d^{2} F}{d \zeta^{2}}\right| \frac{d^{2} F}{d \zeta^{2}}\right)+2 F \frac{d^{2} F}{d \zeta^{2}}=0
$$

while, Görtler [16] assumed

$$
v_{T}=e\left(x-x_{0}\right)
$$

which, with the choice of the self-similarity variable

$$
\xi=\frac{a^{2}\left(\eta-\eta_{0}\right)}{e}
$$

leads to

$$
\frac{d^{3} F}{d \xi^{3}}+F \frac{d^{2} F}{d \xi^{2}}=0
$$

Equations 20 and 23 are solved with the boundary conditions imposed in [15] and [16]. 
Let us now analyze the passive scalar transport in the PCR. From the mean passive scalar budget it is found that, in analogy with the momentum, the time derivative, the molecular diffusion and the axial turbulent diffusion are negligible; therefore, the passive scalar transport equation reduces to

$$
U \frac{\partial \Phi}{\partial x}+V \frac{\partial \Phi}{\partial y}=\frac{\partial}{\partial y}\left(\frac{v_{T}}{S c_{T}} \frac{\partial \Phi}{\partial y}\right)
$$

The turbulent Schmidt number, $S c_{T}$, is normally assumed constant [53], even though some DNS studies show otherwise [40,54]. The measurements in the FDR of a circular heated jet, [55], showed that the turbulent Prandtl number increases near the jet axis and beyond the edge of the jet, regions where the velocity gradient is small in FDR. Further on, a constant value of the passive scalar turbulent diffusivity does not give good predictions in the FDR of a turbulent round jet, may be because the turbulent Schmidt number is not constant across the jet, [6].

The turbulent viscosity and the turbulent Schmidt number are calculated from the present numerical LES results. The turbulent viscosity is defined as the ratio between the mean shear rate and the second invariant of the Reynolds stress tensor. This definition has the advantage of being isotropic, i.e. independent on the flow direction, and is given by

$$
v_{T}=\frac{1}{\bar{\gamma}} \sqrt{\frac{1}{2} \tau_{i j}^{R} \tau_{i j}^{R}-\frac{2}{3} k^{2}}
$$

where $\overline{\dot{\gamma}}$ is the mean shear rate, defined in analogy with the filtered shear rate in Eq. (5), with the mean velocities instead of the filtered ones, $\tau_{i j}^{R}$ is the Reynolds stress tensor and $k$ the turbulent kinetic energy. The turbulent Schmidt number is defined as the ratio between the mean passive scalar gradient and the turbulent passive scalar flux. This definition has the advantage of being isotropic, i.e. independent on the flow direction, and is given by

$$
S c_{T}=v_{T} \sqrt{\frac{\partial \Phi}{\partial x_{j}} \frac{\partial \Phi}{\partial x_{j}}}\left(\sqrt{\overline{\phi^{\prime} u_{k}^{\prime}}} \overline{\phi^{\prime} u_{k}^{\prime}}\right)^{-1}
$$

The turbulent Schmidt number is calculated for all the Reynolds and Schmidt numbers investigated, with the conclusion that is self-similar in the mixing region. Moreover, a relationship between the turbulent Schmidt number and the mean velocity gradient is obtained

$$
S c_{T}=\frac{K_{\Phi}}{\left(x-x_{0}\right)}\left|\frac{\partial U}{\partial y}\right|^{-1}
$$

where the constant $K_{\Phi}$ is equal to 0.8 and independent on the $R e$ and $S c$ numbers. 
The turbulent Schmidt number can be expressed also as a function of both Tollmien, $\zeta$, and Görtler, $\xi$, variables,

$S c_{T}=\alpha_{T}\left|\frac{d^{2} F}{d \zeta^{2}}\right|^{-1}=\alpha_{G}\left|\frac{d^{2} F}{d \xi^{2}}\right|^{-1}$

with the following relationships among $K_{\Phi}$ and the constants $\alpha_{T}, \alpha_{G}$

$K_{\Phi}=\alpha_{G} \frac{a}{e}=\alpha_{T} \frac{1}{\sqrt[3]{2 c^{2}}}$

The turbulent Schmidt number, calculated numerically, is reported in Fig. 17 versus the dimensionless velocity gradient, in the mixing region, only for $S c=1$, because the results are independent from this parameter. The turbulent Schmidt number is inversely proportional to $d U / d \zeta$ (in the logarithmic scale) in the mixing region, hence the passive scalar turbulent diffusivity, i.e. $v_{T} / S c_{T}$, increases with the increasing mean velocity gradient, as the momentum turbulent diffusivity, but with an amplified effect. The diffusion of the passive scalar is greater than the momentum in the mixing region and smaller at the interface with the PCR. It is possible to use the Eq. (28) to produce a simplified theory even in the PCR and at the interface with the stagnant fluid because the velocity and the passive scalar profiles are essentially constant in those regions.
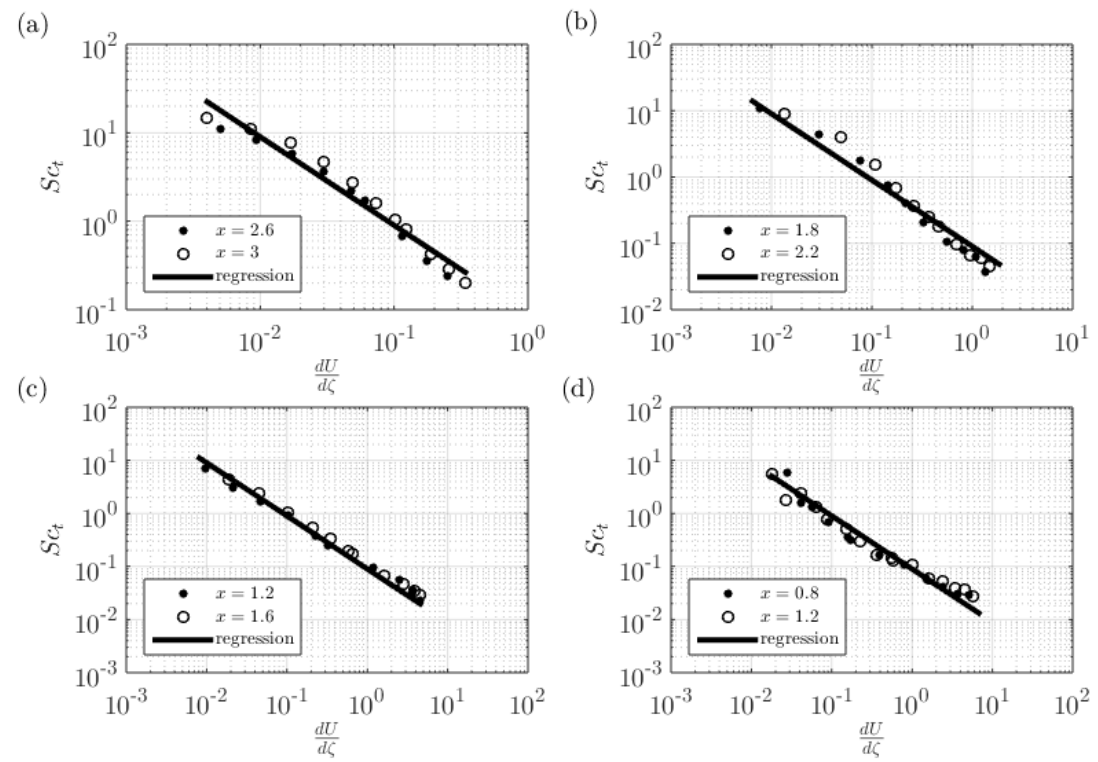

Figure 17: turbulent Schmidt number, numerical and regression, versus dimensionless velocity gradient for $\mathrm{Sc}=1$ in the mixing region. (a) $\operatorname{Re}=5000$; (b) $\operatorname{Re}=10,000 ;$ (c) $\operatorname{Re}=20,000$; (d) $\operatorname{Re}=40,000$.

By using Eq. (28) and the results of Tollmien [15] and Görtler [16] for the momentum, it is possible to derive a "Tollmien-like" solution, which embed the momentum self-similar behavior presented in [15], for the passive scalar transport 
$\frac{d}{d \zeta}\left(\left|\frac{d^{2} F}{d \zeta^{2}}\right|^{2} \frac{d \Phi}{d \zeta}\right)+2 \alpha_{T} F \frac{d \Phi}{d \zeta}=0$

and, similarly, a "Görtler -like" solution, which embed the momentum self-similar behavior presented in [16], for the passive scalar transport

$\frac{d}{d \xi}\left(\left|\frac{d^{2} F}{d \xi^{2}}\right| \frac{d \Phi}{d \xi}\right)+\alpha_{G} F \frac{d \Phi}{d \xi}=0$

The previous two equations are solved with the following boundary conditions

$$
\begin{aligned}
& \lim _{\zeta \rightarrow+\infty} \Phi(\zeta)=\lim _{\xi \rightarrow+\infty} \Phi(\xi)=0 \\
& \lim _{\zeta \rightarrow-\infty} \Phi(\zeta)=\lim _{\xi \rightarrow-\infty} \Phi(\xi)=1
\end{aligned}
$$

obtaining the self-similar mean passive scalar profile, following Tollmien's behavior [15],

$$
\Phi(\zeta)=1-\frac{\int_{-\infty}^{\zeta}\left|\frac{d^{2} F}{d \eta^{2}}\right|^{-2} \exp \left(-2 \alpha_{T}\left|\frac{d^{2} F}{d \eta^{2}}\right|^{-1}\right) d \eta}{\int_{-\infty}^{+\infty}\left|\frac{d^{2} F}{d \eta^{2}}\right|^{-2} \exp \left(-2 \alpha_{T}\left|\frac{d^{2} F}{d \eta^{2}}\right|^{-1}\right) d \eta}
$$

and the self-similar mean passive scalar profile, following Görtler's behavior [16],

$$
\Phi(\xi)=1-\frac{\int_{-\infty}^{\xi}\left|\frac{d^{2} F}{d \eta^{2}}\right|^{-1} \exp \left(-\alpha_{G}\left|\frac{d^{2} F}{d \eta^{2}}\right|^{-1}\right) d \eta}{\int_{-\infty}^{+\infty}\left|\frac{d^{2} F}{d \eta^{2}}\right|^{-1} \exp \left(-\alpha_{G}\left|\frac{d^{2} F}{d \eta^{2}}\right|^{-1}\right) d \eta}
$$

The mass transfer per unit area in the cross-stream direction is function of the local turbulent Schmidt number and increases with the velocity gradient, although without a direct proportionality.

$$
\left|J_{y}\right|=\frac{v_{T}}{S c_{T}}\left|\frac{\partial \Phi}{\partial y}\right|=\frac{\frac{1}{2 K_{\Phi}} \exp \left(-2 S c_{T}\right)}{\int_{-\infty}^{+\infty}\left|\frac{d^{2} F}{d \eta^{2}}\right|^{-2} \exp \left(-2 \alpha_{T}\left|\frac{d^{2} F}{d \eta^{2}}\right|^{-1}\right) d \eta}
$$




$$
\left|J_{y}\right|=\frac{v_{T}}{S c_{T}}\left|\frac{\partial \Phi}{\partial y}\right|=\frac{\frac{1}{K_{\Phi}} \frac{a^{2}}{e} \exp \left(-S c_{T}\right)}{\int_{-\infty}^{+\infty}\left|\frac{d^{2} F}{d \eta^{2}}\right|^{-1} \exp \left(-\alpha_{G}\left|\frac{d^{2} F}{d \eta^{2}}\right|^{-1}\right) d \eta}
$$

The self-similar mean passive scalar profiles, shown in terms of the Tollmien self-similar variable $\zeta$ and obtained from the proposed theories, are reported in Figs. 18-19, at the four Reynolds numbers investigated and for Schmidt number equal to 1 and 100 respectively. The theoretical profiles are compared with the LES numerical results at several axial distances, in order to show that the hypothesis of self-similarity is verified. The self-similar mean passive scalar profiles give comparable results at the interface, with a better agreement with the Tollmien theory [15] close to the centerline, and with the Görtler one [16] in the outer region, as already found for the momentum in [36-37]. The agreement among the numerical results and the two proposed theories is quite good, even at high Reynolds numbers. The mean passive scalar profile shows an inflection point at $\Phi \approx 0.8945$, which corresponds to $\left|d^{2} F / d \zeta^{2}\right|=\alpha_{T}$, or $\left|d^{2} F / d \xi^{2}\right|=\alpha_{G}$, or, by virtue of Eq. (28), to $S c_{T}=1$. At the interface between the PCR and the mixing region, the velocity gradient is very small, and therefore $S c_{T}>1$, which implies that the passive scalar diffusion is smaller than the momentum. When the velocity gradient assumes the critical value corresponding to $S c_{T}=1$, the passive scalar diffuses as fast as the momentum. At the center of the mixing region, the velocity gradient grows and $S c_{T}<1$, hence the passive scalar diffuses faster than the momentum. Another slope change is observable for $\Phi \approx 0.2682$, where the passive scalar penetrates into the stagnant fluid.

The variable Schmidt number is the reason for the slope change in the LES results. Indeed, comparing the mean passive scalar profiles with the velocity ones, found in [36-37], it is confirmed that the two profiles have a different shapes because of the variable Schmidt number. This conclusion is different from what found in the FDR of circular jets, where the passive scalar is proportional to the axial velocity through a power-law relationship with exponent $S c_{T}$ [6-7]. 
(a)

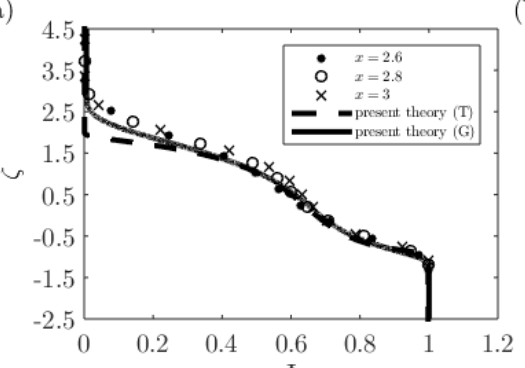

(c)

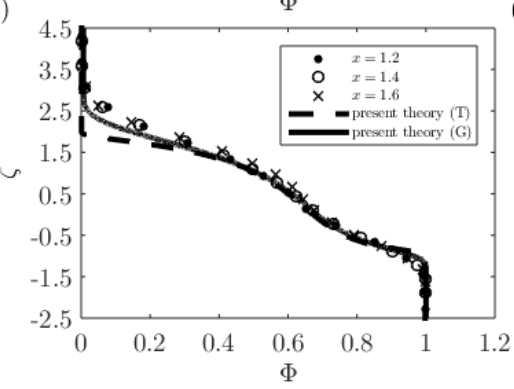

(b)

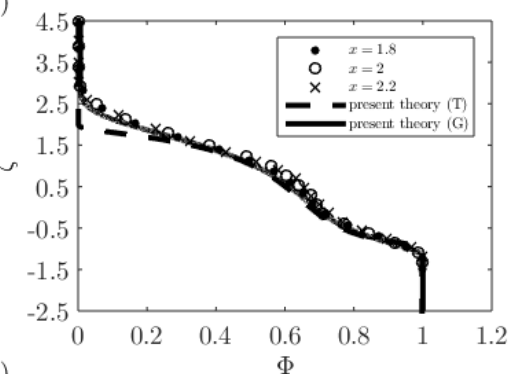

(d)

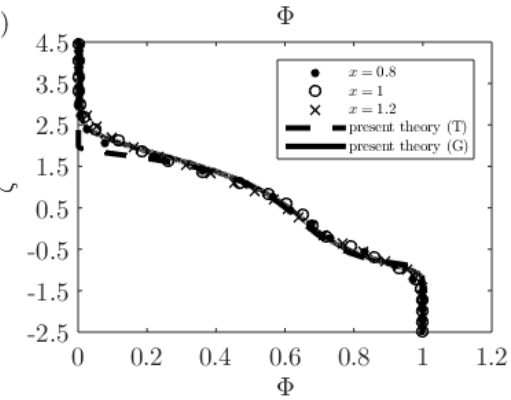

Figure 18: passive-scalar profile in PCR for Sc=1. Numerical and "Tollmien-like" and "Görtler -like" theoretical results. (a) $\operatorname{Re}=5,000$; (b) $\operatorname{Re}=10,000$; (c) $\operatorname{Re}=20,000$; (d) $\operatorname{Re}=40,000$.

(a)

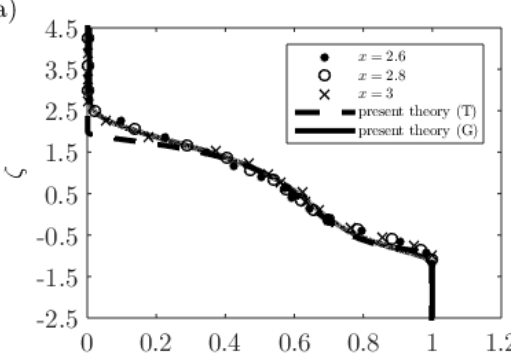

(c)

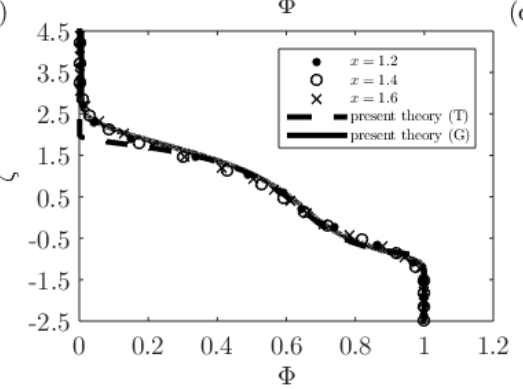

(b)

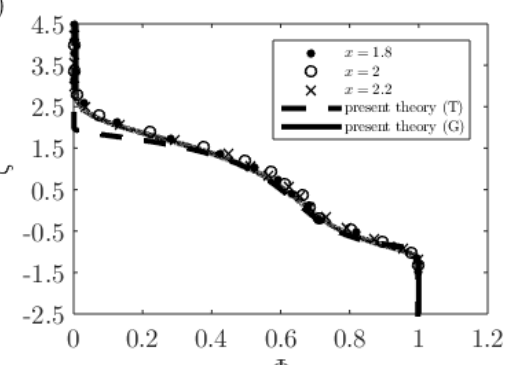

(d)

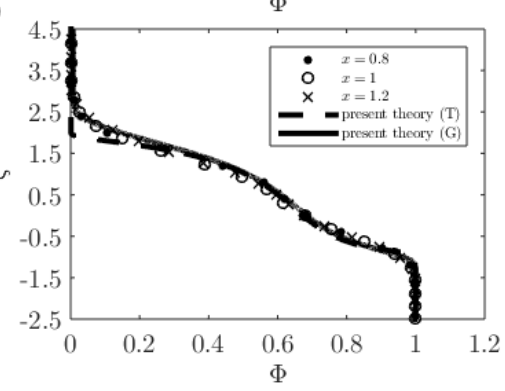

Figure 19: passive-scalar profile in PCR for $\mathrm{Sc}=100$. Numerical and "Tollmien-like" and "Görtler -like" theoretical results. (a) $\operatorname{Re}=5,000 ;$ (b) $\operatorname{Re}=10,000$; (c) $\operatorname{Re}=20,000$; (d) $\operatorname{Re}=40,000$.

\section{Conclusions}

A series of Large Eddy Simulations (LES) are performed at four Reynolds numbers, namely 5000, 10,000, 20,000 and 40,000, to investigate the passive scalar spreading in the Near Field Region (NFR) of turbulent submerged jets, issuing from a rectangular slot. Because the particles (or droplets) laden in the air jet can have variable dimensions, and therefore variable molecular diffusivities, two Schmidt numbers, namely 1 and 100 , are investigated in order to have a model of large applicability at the largest Peclet number of $4 \cdot 10^{6}$. 
The results of the present paper confirm that the passive scalar profile, in analogy with the axial velocity, is self-similar also in the NFR, which, in the mean flow, is divided in two regions, the Undisturbed Region of Flow (URF) and the Potential Core Region (PCR). The passive scalar is shown to be function of the self-similar variables introduced in [36-37] for the URF. A self-similar law is proposed for the evolution of the passive scalar and the theoretical results are compared with the numerical ones, showing a good agreement. The mean passive scalar profiles are function of the self-similar variables introduced in [15-16]. The LES results confirmed that the turbulent Schmidt number is a self-similar variable in the mixing region, and is inversely proportional to the axial velocity gradient. The numerical results are compared to the mathematical model with a good agreement.

The present results show that turbulence affects in a different way the momentum and the passive scalar transport. The passive scalar diffuses more, compared to the momentum, in the mixing region, since the turbulent Schmidt number is smaller than 1. This conclusion can explain why the coherent structures are observable when a tracer is introduce in a jet, i.e. in PIV investigations [29-34], while they are not observable from the velocity raw data [56]. The present work gives an analytical tool to estimate the spreading of a passive scalar in the NFR of a rectangular submerged jet.

\section{References}

[1] I.V. Derevich, Influence of internal turbulent structure on intensity of velocity and temperature fluctuations of particles, International journal of heat and mass transfer, 44 (23) (2001) 4505-4521.

[2] J.S. Wu, Y.J. Liu, H.J. Sheen, Effects of ambient turbulence and fuel properties on the evaporation rate of single droplets, International journal of heat and mass transfer, 44 (24) (2001) 4593-4603.

[3] J.J. Nijdam, T.A.G. Langrish, D.F. Fletcher, Assessment of an Eulerian CFD model for prediction of dilute droplet dispersion in a turbulent jet, Applied Mathematical Modelling 32 (12) (2008) 2686-2705.

[4] F. Gori, I. Petracci, Influence of turbulence on heat transfer upon a cylinder impinged by a slot jet of air, Applied Thermal Engineering, 49 (2012) 106-117.

[5] F. Gori, I. Petracci, Influence of screen solidity ratio on heat transfer upon a cylinder impinged by a rectangular jet, International Journal of Heat and Mass Transfer, 81 (2015) 19-27.

[6] J.O. Hinze, Turbulence, McGraw-Hill, New York (1975).

[7] A.A. Townsend, The structure of turbulent shear flow, Cambridge university press, 1980.

[8] K. Chen, L. Miao, A Lorentz Force Powered Controllable Micro-Jet Injection System, in: Computer Science and Engineering Technology (CSET2015), Medical Science and Biological Engineering (MSBE2015), Proceedings of the 2015 International Conference on CSET and MSBE, World Scientific, 2015, p. 170. 
[9] J.J. Yoh, H. Jang, M. Park, T. Han, J. Hah, A bio-ballistic micro-jet for drug injection into animal skin using a Nd: YAG laser, Shock Waves, 26 (1) (2016) 39-43.

[10] D. Hadryś, T. Węgrzyn, J. Piwnik, Ł. Wszołek, D. Węgrzyn, Compressive Strength of Steel Frames after Welding with Micro-Jet Cooling, Archives of Metallurgy and Materials, 61 (1) (2016) 123-126.

[11] T. Wegrzyn, J. Piwnik, B. Lazarz, W. Tarasiuk, Mechanical properties of shaft surfacing with micro-jet cooling, Mechanics, 21 (5) (2015) 419-423.

[12] M.A. Islam, Einstein-Smoluchowski diffusion equation: a discussion, Physica Scripta, 70 (2-3) (2004) 120.

[13] E. Cunningham, On the velocity of steady fall of spherical particles through fluid medium, Proceedings of the Royal Society of London, Series A, Containing Papers of a Mathematical and Physical Character, 83 (563) (1910) 357-365.

[14] M.L. Albertson, Y.B. Dai, R.A. Jensen, H. Rouse, Diffusion of Submerged Jets, Trans. Am. Soc. Civ. Eng., 115 (1) (1950) 639-64.

[15] W. Tollmien, Calculation of Turbulent Expansion Processes, Zeitschrift Für Angewandte Mathematik Und Mechanik, 6 (6) (1926) 468-78.

[16] H. Görtler, Berechnung von Aufgaben Der Freien Turbulenz Auf Grund Eines Neuen Naherungsansatzes, Zeitschrift Für Angewandte Mathematik Und Mechanik, 22 (5) (1942) 244-254.

[17] E. Forthmann, Turbulent Jet Expansion, NACA-TM-789, 1936.

[18] D.R. Miller, E.W. Comings, Static Pressure Distribution in the Free Turbulent Jet, J. Fluid Mech., 3 (1) (1957) 1-16.

[19] B.G. Van der Hegge Zijnen, Measurements of the Distribution of Heat and Matter in a Plane Turbulent Jet of Air, Appl. Sci. Res., 7 (4) (1958) 277-292.

[20] H.W. Liepmann, J. Laufer, Investigations of Free Turbulent Mixing, NACA Technical Note, 1947.

[21] F.O. Thomas, V.W. Goldschmidt, Structural Characteristics of a Developing Turbulent Planar Jet, J. Fluid Mech. 163 (1986) 227-256.

[22] L.J.S. Bradbury, The Structure of a Self-Preserving Turbulent Plane Jet, Journal of Fluid Mechanics, 23 (01) (1965) 31-64.

[23] E. Gutmark, I. Wygnanski, The Planar Turbulent Jet, Journal of Fluid Mechanics, 73 (3) (1976). 465.

[24] A.A. Sfeir, The Velocity and Temperature Fields of Rectangular Jets, International Journal of Heat and Mass Transfer, 19 (11) (1976) 1289-97. 
[25] A. Krothapalli, D. Baganoff, K. Karamcheti, On the Mixing of a Rectangular Jet, Journal of Fluid Mechanics, 107 (1981) 201-20.

[26] G.B. Brown, On Vortex Motion in Gaseous Jets and the Origin of Their Sensitivity to Sound, Proc. Phys. Soc., 47 (1935) 703.

[27] G.S. Beavers, T.A. Wilson, Vortex Growth in Jets, J. Fluid Mech., 44 (1970) 97-112.

[28] A. Chambers, R. Antonia, L. Browne, Effect of Symmetry and Asymmetry of Turbulent Structures on the Interaction Region of a Plane Jet, Exp. Fluids, 3 (1985) 343-348.

[29] F. Gori, F. De Nigris, E. Nino, Fluid Dynamics Measurements and Optical Visualization of the Evolution of a Submerged Slot Jet of Air, Proceedings of the 12th International Heat Transfer Conference, Grenoble (France), Volume 2, 303-308, August 2002, Heat Transfer 2002. Editions Scientifique et Medicales, Elsevier SAS.

[30] F. Gori, I. Petracci, Fluid dynamics measurements and numerical simulations around a circular cylinder impinged by a submerged slot jet of air, ASME 2003 International Mechanical Engineering Congress and Exposition, American Society of Mechanical Engineers, (2003) 179-185.

[31] F. Gori, I. Petracci, Fluid Dynamics Measurements in a Submerged Free Slot Jet of Air, 14th International Symposium on Transport Phenomena, Bali, Indonesia, (2003) 491-496.

[32] F. Gori, E. Nino, Fluid Dynamics Measurements and Flow Visualizations of a Free Slot Jet of Air, American Society of Mechanical Engineers, Fluids Engineering Division (Publication) FED, 259 (2003) 187192, ASME International Mechanical Engineering Congress; Washington, DC, United States; 15 November 2003 through 21 November 2003; Code 62622.

[33] F. Gori, E. Nino, I. Petracci, Shadowgraph Visualizations of a Submerged Free Slot Jet of Air, Int. J. Heat Technology, 25 (1) (2007) 157-164.

[34] F. Gori, I. Petracci, M. Angelino, Flow Evolution of a Turbulent Submerged Two-Dimensional Rectangular Free Jet of Air. Average Particle Image Velocimetry (PIV) Visualizations and Measurements, Int. J. Heat Fluid Flow, 44 (2013) 764-775.

[35] F. Gori, M. Angelino, A. Boghi, I. Petracci, Preliminary Numerical Solutions of the Evolution of Free Jets, ASME International Mechanical Engineering Congress and Exposition, Proceedings (IMECE), Volume 7, Issue PARTS A, B, C, D, 2012, Pages 463-469, ASME 2012 International Mechanical Engineering Congress and Exposition, IMECE 2012; Houston, TX; United States, 9 November 2012 through 15 November 2012; Code 100737.

[36] A. Boghi, M. Angelino, F. Gori, Numerical evidence of an undisturbed region of flow in a turbulent rectangular submerged free jet, Numerical Heat Transfer, Part A: Applications, 70 (1) (2016): 14-29. 
[37] M. Angelino, A. Boghi, F. Gori, Numerical solution of three-dimensional rectangular submerged jets with evidence of the undisturbed region of flow, Numerical Heat Transfer, Part A: Applications, 70 (8) (2016) 815830.

[38] R. Kumar, A. Dewan, URANS computations with buoyancy corrected turbulence models for turbulent thermal plume, International Journal of Heat and Mass Transfer, 72 (2014) 680 - 689.

[39] S.N. Riddick, B.R. Hancock, A.D. Robinson, S. Connors, S. Davies, G. Allen, J. Pitt, N.R.P. Harris, Development of a low-maintenance measurement approach to continuously estimate methane emissions: A case study, Waste Management, (2016, in press).

[40] F. Schwertfirm, M. Manhart, DNS of passive scalar transport in turbulent channel flow at high Schmidt numbers, International Journal of Heat and Fluid Flow, 28 (6) (2007) 1204-1214.

[41] Z. Wang, P. He, Y. Lv, J. Zhou, J. Fan, K. Cen, Direct numerical simulation of subsonic round turbulent jet, Flow, turbulence and combustion, 84 (4) (2010) 669-686.

[42] S. Stanley, S. Sarkar, Simulations of Spatially Developing Two-Dimensional Shear Layers and Jets, Theoretical and Computational Fluid Dynamics, 9 (2) (1997) 121-47.

[43] K. Luo, J. Yan, J. Fan, K. Cen, On coherent structures in a three-dimensional transitional plane jet, Science in China Series E: Technological Sciences, 51 (4) (2008) 386-396.

[44] J. Fan, K. Luo, M.Y. Ha, K. Cen, Direct numerical simulation of a near-field particle-laden plane turbulent jet, Physical Review E., 70 (2) (2004) 026303.

[45] P.E. DesJardin, S.H. Frankel, Large eddy simulation of a nonpremixed reacting jet: Application and assessment of subgrid-scale combustion models, Physics of Fluids, 10 (9) (1998) 2298-2314.

[46] S. James, F.A. Jaberi, Large Scale Simulations of Two-Dimensional Nonpremixed Methane Jet Flames, Combustion and Flame, 123 (2000) 465-487.

[47] Y. Liu, K.S. Lau, C.K. Chan, Y.C. Guo, W.Y. Lin, Structures of Scalar Transport in 2D Transitional Jet Diffusion Flames by LES, International Journal of Heat and Mass Transfer, 46 (20) (2003) 3841-51.

[48] Y. Liu, P.G. Tucker, R.M. Kerr, Linear and nonlinear model large-eddy simulations of a plane jet, Computers \& Fluids, 37 (4) (2008) 439-449.

[49] M. Lesieur, Turbulence in Fluids, Kluwer Academic, Boston, MA, USA, 1990.

[50] P. Moin, K. Squires, W. Cabot, S. Lee, A dynamic sub-grid-scale model for compressible turbulence and scalar transport, Phys. Fluids. 3 (1991) 2746.

[51] F. Gori, I. Petracci, M. Angelino, Influence of the Reynolds number on the instant flow evolution of a turbulent rectangular free jet of air, International Journal of Heat and Fluid Flow, 50 (2014) 386-401. 
[52] S. Chandrasekhar, Hydrodynamic and Hydromagnetic Stability, Clarendon Press: Oxford University Press, 1961.

[53] Y. Tominaga, T. Stathopoulos, Turbulent Schmidt numbers for CFD analysis with various types of flowfield, Atmospheric Environment, 41 (37) (2007) 8091-8099.

[54] M. van Reeuwijk, M. Hadžiabdić, Modelling high Schmidt number turbulent mass transfer, International Journal of Heat and Fluid Flow, 51 (2015) 42-49.

[55] L.P Chua, and R.A. Antonia, Turbulent Prandtl number in a circular jet, International Journal of Heat and Mass Transfer, 33 (2) (1990) 331-339.

[56] F. Gallaire, S. Rott, J.M. Chomaz, Experimental study of a free and forced swirling jet, Physics of Fluids, 16 (8) (2004) 2907-2917. 